\title{
Optimal sequestration policy with a ceiling on the stock of carbon in the atmosphere
}

\author{
Gilles Lafforgue*, Bertrand Magné ${ }^{\dagger}$ \\ and \\ Michel Moreaux $\S^{\ddagger}$
}

\footnotetext{
${ }^{*}$ Université de Toulouse 1 (INRA and LERNA), 21 Allée de Brienne, 31000 Toulouse, France. E-mail: glafforg@toulouse.inra.fr.

${ }^{\dagger}$ Université de Toulouse 1 (CEA and LERNA), 21 Allée de Brienne, 31000 Toulouse, France. E-mail: bertrand.magne@univ-tlse1.fr.

${ }^{\ddagger}$ Université de Toulouse I (IUF, IDEI and LERNA), 21 Allée de Brienne, 31000 Toulouse, France. E-mail: moreaux@cict.fr.

$\S$ The authors wish to thank an anonymous referee for providing valuable comments.
} 


\section{Contents}

1 Introduction $\quad 2$

2 The model $\quad 6$

2.1 Assumptions and notations . . . . . . . . . . . . . . . 6

2.2 Problem formulation . . . . . . . . . . . . . . . . . . . . . . 10

3 Hotelling and optimal paths without any carbon sink 12

3.1 Pure Hotelling paths . . . . . . . . . . . . . . . . . . . . . . . . . . . . . . 12

3.2 Optimal paths with no active abatement opportunity . . . . . . . . . . 13

4 The case of an abundant renewable substitute $\quad 15$

4.1 Testing the optimality of the sequestration opportunity . . . . . . . . . . . 15

4.2 The large reservoir case . . . . . . . . . . . . . . . . . . . . . 16

4.3 The small reservoir case . . . . . . . . . . . . . . . . . . . . . . . 19

5 The case of a rare renewable substitute $\quad 20$

5.1 Testing the optimality of the sequestration opportunity . . . . . . . . . . . . 20

5.2 Optimal paths for beginning sequestration before using the renewable sub-

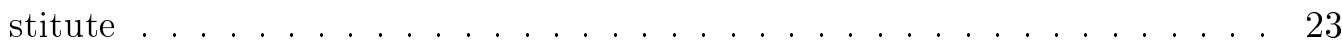

5.3 Optimal paths for beginning use of the renewable substitute before sequestration . . . . . . . . . . . . . . . . . . . . . . 24

6 Conclusion 25

$\begin{array}{ll}\text { Appendix } & 27\end{array}$

$\begin{array}{ll}\text { References } & \mathbf{2 9}\end{array}$ 


\section{Introduction}

We determine the optimal exploitation time-paths of two energy resources, one being depletable and carbon-based, i.e. polluting with regard to climate change, namely a fossil fuel (coal, oil or gas), the other being renewable, clean and non-biological ${ }^{1}$ (e.g. solar energy directly converted via photovoltaic cells, or indirectly converted as in the case of wind energy). These optimal paths are considered along with the two following features. First, the cumulative atmospheric pollution stock is set not to exceed some critical threshold, above which the induced environmental damage cost would be unbearable. Second, the pollutant emissions produced by the use of fossil fuel use can be reduced at source, and stockpiled in a natural reservoir, referred to here as a carbon sink ${ }^{2}$.

In its latest report (IPCC, 2005), the IPCC recommends recourse to the capture and sequestration of carbon (CCS) to reduce anthropogenic $\mathrm{CO}_{2}$ emissions to the atmosphere. CCS consists in filtering the $\mathrm{CO}_{2}$ fluxes at the source of emission, that is, in fossil energyfuelled power plants, by means of scrubbers installed on top of chimney stacks. Carbon may be sequestered in geological reservoirs, among which are discussed coal mines, depleted oil and gas reservoirs ${ }^{3}$, as well as deep saline aquifers, and even the ocean itself (IPCC, 2005). The respective potential capacities of these carbon sinks as well as their efficiency are still under assessment ${ }^{4}$.

To our knowledge, there are no analytical studies that clearly demonstrate the tradeoffs between the management of an exhaustible fossil resource, the accumulation of the related pollution and sequestration of limited capacity. Nonetheless, the possibility of sequestering some fraction of the pollution has motivated a number of empirical studies, via complex integrated assessment models (see Mc-Farland et al. 2003; Edmonds et al. 2004; Kurosawa 2004; Gitz et al. 2005). These studies generally stress that the implementation of sequestration leads to a substantial decrease in the cost of environmental externality, which thus favor the early introduction of such measures.

\footnotetext{
${ }^{1}$ See Chakravorty et al. (2005a) for the specificities of the substitutions between fossil and bio-energies.

${ }^{2}$ A carbon sink sometimes refers to biological sequestration, that is, carbon captured by photosynthesis of trees or plants. This is not the focus of the present paper.

${ }^{3}$ Carbon sequestration in partially or totally depleted oil deposits has been carried out in the North Sea since 1996 by the Norwegian company Statoil. The enhanced oil recovery consists in injecting gas into the oil well, thus increasing pressure and improving the extraction productivity.

${ }^{4}$ Oceanic storage, despite its enormous potential as a sink, comprises several limitations including the issue of sequestration permanence and problems of acceptability since it may also lead to toxicity risks due to water acidification and threats to marine ecosystems (See Herzog et al., 2003).
} 
Such a generic abatement option can take several forms: sequestration by forests, pollution reduction at source, etc. In this study, we are only concerned with the capture and direct disposal of carbon, while taking into consideration the size and access cost of the reservoir.

We introduce four features into the standard Hotelling model of exhaustion. The first is the possibility of providing the economy with a cleaner backstop in the form of an abundant energy flow. Following an optimal path in such an economy, the resource price has two components: its marginal extraction cost and the Hotelling rent, which necessarily grows at a rate equal to the interest rate and which drives the prices up over time. When this price reaches the marginal cost of the backstop, which is assumed to be constant, only the renewable source is used. Thus, the two energy sources are used one after the other and the backstop is only introduced when the fossil resource has been exhausted.

The second feature is the ceiling placed on cumulative carbon emissions from the consumption of a fossil energy resource. The changes in its consumption drive the dynamics of pollution accumulation as well as the ultimate transition to a cleaner technology, since each extraction trajectory generates a cumulative emission trajectory. This ceiling constraint adds a third component to the expression of the fossil fuel price: the externality cost associated with the accumulation of pollution in the atmosphere. In this context, it is crucial that there should be a certain amount of natural regeneration reducing the level of pollution, thus allowing some use of the fossil fuel to continue whilst at the ceiling. We then show that the optimal consumption path consists of four phases. Firstly, only the fossil fuel resource supplies the economy. During this phase, the scarcity rent of the resource and the shadow cost of carbon emissions are both increasing. Hence, the energy price increases and the fossil fuel consumption (and hence the emission flow) decreases, assuming that the demand function is stationary over time ${ }^{5}$. However, the amount of pollutants in the atmosphere increases because the emission flow is larger than the regenerated flow. Once the ceiling is attained, the fossil fuel consumption is limited by the natural regeneration process. When the pollution stock is at its ceiling level - as induced by natural regeneration flow - it is just in balance with the emission flow. Fossil fuel consumption and energy price both remain constant. Because of the increasing Hotelling rent (in current value), the shadow cost of the constraint on carbon emissions must decrease during this

\footnotetext{
${ }^{5}$ For the much more complex case of non-stationary demand functions, see Chakravorty et al. (2005b).
} 
phase at the ceiling. This second phase ends when the shadow cost finally drops to zero. During the third phase, the price increases once again, driven only by the increase in the scarcity rent, rising until it is equal to the marginal cost of the backstop and the fossil fuel becomes exhausted. Meanwhile, emissions are decreasing, the ceiling constraint is no longer limiting and the path reverts to the benchmark Hotelling level. Lastly, during the fourth phase, the backstop supplies the whole demand. This scheme holds as long as the energy price, defined at the ceiling when only fossil fuel is used, is higher than the marginal cost of the clean substitute. If this marginal cost is lower and the clean resource is abundant, the clean energy would be substituted for the fossil fuel at the precise time when the ceiling is attained. At that time, its full marginal cost (i.e. the sum of the marginal extraction cost, the Hotelling rent and the shadow cost of the carbon constraint) must be equal to the marginal cost of the clean technology. At the ceiling, emissions are balanced by the regeneration process, some part of the supply is provided by the renewable resource and the energy price is equal to the marginal cost of the renewable resource as shown in Chakravorty et al. (2004). Both resources have to be exploited simultaneously because, at this price, even if the renewable is competitive, the fossil resource remains less costly (excluding the cost of externality) and thus has to be used jointly. At the ceiling, the fossil fuel supply is indirectly restricted by the regeneration flow. During this phase, the increase of the Hotelling rent is compensated by the decrease of the (positive) shadow cost of the carbon ceiling, so that the energy price is constant and equal to the marginal cost of the renewable energy. On complete exhaustion of the fossil fuel, the shadow cost of carbon becomes nil and the renewable resource supplies the entire demand. In this case of lowcost substitute, there is no longer any pure Hotelling phase.

The third feature corresponds to the capture and storage of some fraction of the carbon emissions. This carbon sequestration could leave room for the continued intensive use of fossil fuels by alleviating the environmental consequences of their combustion, which are especially implicated in the climate change process. Such a mitigation option would also lead to less stringent Kyoto-type constraints on greenhouse gases emissions, even if, as discussed below, the optimal policy may not be consistent with the type of policy laid down by the Protocol.

However, the alleviation option is not available free of charge. To sequester carbon incurs some additional costs depending on the characteristics of the sink, especially its 
size. Chakravorty et al. (2005b) evoke a generic abatement activity in which carbon sinks are of unlimited capacity. This simply implies that an emission processing cost component should be considered when determining the resource price. More interesting is the case where the capacity of the carbon sink is limited. The marginal cost of consuming one unit of fossil fuel compatible with some environmental preservation objective is thus four-fold: it includes the monetary cost of exploiting the resource, the cost of carbon processing, the scarcity rent of the resource and the rent associated with the limited capacity of the carbon sink, both rents being endogenous. This overall cost needs to be compared with the supply cost of the renewable energy, this cost being generally higher than the exploitation cost of the fossil fuel alone. If the cost of the renewable energy is higher than the sum of the cost of fossil resource exploitation and the cost of carbon sequestration, then it is better to exploit the depletable resource before the renewable one. We then show how the application of a capture option at the source of pollution emission leads to earlier competitiveness of the clean substitute. Carbon sequestration thus relaxes the constraints on fossil fuel consumption. An immediate implication is that the non-renewable resource is exhausted earlier, and the renewable one kicks in earlier. Finally, the optimal path is shown to consist of five phases. As long as the ceiling is not reached, only the fossil resource is used and the pollution stock continues to increase. Once the ceiling is attained, carbon sequestration takes place until complete filling of the sink. The next phase occurs at the ceiling without sequestration. The two following phases are identical to the three last phases that occur in the case of a pollution stock ceiling without any sequestration.

Let us assume that the clean renewable substitute is scarce, meaning that, at a price equal to its marginal cost, the market demand is greater than the available flow. In this case, even without any pollution constraint, the resources are no longer exploited sequentially. Once the fossil fuel price (i.e. the sum of the extraction cost and the Hotelling rent) is equal to the marginal cost of the renewable substitute, renewable energy becomes competitive and has to be exploited. But since the available flow cannot satisfy the whole demand, in order to clear the market, the residual demand must be supplied by the fossil resource. During this phase of simultaneous use of both resources, prices should vary according to the same rule as in the first phase because the scarcity rent of the fossil is still growing at the interest rate. Thus, the discrepancy between the energy price and the marginal cost of the renewable resource increases at a rate higher than the interest rate. 
This is a consequence of the non-storability of the resource, excluding any intertemporal arbitrage, which allows the rent of the renewable resource to grow faster than the rise in interest rate. The share of consumption supplied by the fossil fuel decreases continuously until complete exhaustion of the resource. The backstop finally supplies the whole demand. When a cap is set on carbon accumulation, the use of the clean renewable substitute begins before the pollution ceiling is reached provided the energy price at that time is higher than the marginal cost of the substitute. By the same token, use of the substitute will begin after pollution reaches the ceiling if the energy price is lower than the marginal cost of the substitute. Furthermore, if there is an opportunity for sequestration, whether it is applied before or after using the renewable resource depends upon their respective costs.

The paper has the following structure. In section 2, we present the general formulation of the model. We then describe in section 3 the characteristics of the solution when carbon sequestration is not an option. In section 4 , we provide a simple test to check the optimality of the sequestration option, and then determine the optimal sequestration policy depending on whether the size of the reservoir is large or small, this property being endogenous in the sense defined in this study. In section 5, we analyze the implications of a limited availability of the renewable resource, which may be alternatively expensive or cheap in relation to the price of energy compatible with maintaining a pollution ceiling. We conclude briefly in section 6 .

\section{The model}

\subsection{Assumptions and notations}

We consider an economy in which the instantaneous gross surplus or utility, measured in monetary units and generated by an instantaneous energy consumption $q_{t}{ }^{6}$, is given by the following standard function $u$.

Assumption A.1 $: u: \mathbb{R}_{++} \rightarrow \mathbb{R}_{+}$is a function of class $\mathcal{C}^{2}$ strictly increasing and strictly concave satisfying the Inada condition, i.e. $\lim _{q \downarrow 0} u^{\prime}(q)=+\infty$, where $u^{\prime}(q)=d u / d q$.

\footnotetext{
${ }^{6}$ Strictly speaking, $q_{t}$ is a power, so assuming that $q_{t}$ is differentiable, the energy consumed over a time interval $[t, t+d t]$ is equal to $\left(q_{t}+\dot{q}_{t}\right) d t$, where $\dot{q}_{t}=d q_{t} / d t$.
} 
We sometimes use $p$ to denote the marginal surplus $u^{\prime}$ as well as (by a slight misuse of formal notation) the marginal surplus function: $p(q)=u^{\prime}(q)$. The direct demand function $d(p)$ is merely the reciprocal of $p(q)$, as usually defined.

Energy needs may be supplied by two resources, either a dirty non-renewable resource, such as coal, or a clean renewable resource, such as solar energy. If $X^{0}$ represents the initial coal stock of the society, $X_{t}$ the stock of coal available at time $t\left(X_{0}=X^{0}\right)$ and $x_{t}$ the instantaneous coal consumption, we can write:

$$
\dot{X}_{t}=-x_{t}
$$

We assume that the average cost of transforming coal into energy directly usable by the final users is constant and equal to $c_{x}$, hence $c_{x}$ is also the constant marginal cost. This cost should be regarded as the sum of the extraction cost sensu stricto, the cost of industrial processing of the extraction output and the cost of transportation, all of which must be borne so the energy supply can match the demand by end users.

Let $\tilde{x}$ denote the flow of non-renewable resource to be consumed, assuming an infinite available stock of the non-renewable resource $X^{0}$, so that no rent would have to be charged. Thus, $\tilde{x}$ is the solution of $u^{\prime}(x)=c_{x}$, that is, $\tilde{x}=d\left(c_{x}\right)$.

Using coal potentially generates a pollutant flow. Let $\zeta$ be the unitary carbon content of coal so that, without any abatement policy, the instantaneous carbon flow released into the atmosphere would be equal to $\zeta x_{t}$. However, let us assume that some carbon sequestration device is available. Let $s_{t}$ be the part of the potential carbon emission flow that is sequestered, so that the effective flow, denoted by $z_{t}$, amounts to:

$$
z_{t}=\zeta x_{t}-s_{t} \quad \text { with } \quad s_{t} \geq 0 \quad \text { and } \quad \zeta x_{t}-s_{t} \geq 0 .
$$

We assume that the unit sequestration cost is constant (hence also the marginal cost) and equal to $c_{s}$, so that the total monetary cost of sequestration is given by $c_{s} s_{t}$. $\bar{S}$ denotes the capacity of the so-called carbon sink, $S^{0}$ the initial stock of carbon contained in the sink and $S_{t}$ the stock at time $t\left(S_{0}=S^{0}\right)$, we can write:

$$
\dot{S}_{t}=s_{t} \quad \text { and } \quad \bar{S}-S_{t} \geq 0
$$

Without any loss of generality, we postulate that $S^{0}=0$. 
Let $Z^{0}$ be the stock of carbon in the atmosphere at the beginning of the planning period, $Z_{t}$ the stock at time $t\left(Z_{0}=Z^{0}\right)$ and $\alpha(>0)$ the instantaneous proportional rate of natural regeneration, assumed to be constant for sake of simplicity (see Kolstad and Krautkraemer, 1993 ${ }^{7}$ so that:

$$
\dot{Z}_{t}=z_{t}-\alpha Z_{t}
$$

Self regeneration is merely a scheme for natural sequestration into a sink of sufficiently large capacity. By that, we mean that, whatever the quantity of carbon needing to be sequestered, it still can still be buried in the so-called sink.

We assume that this stock of carbon cannot be larger than some threshold $\bar{Z}$ :

$$
\bar{Z}-Z_{t} \geq 0 \quad \text { and } \quad \bar{Z}-Z^{0} \geq 0 \text {. }
$$

This constraint should be considered as some kind of damage function. The damage generated at each point of time by the stock of atmospheric carbon is equal to 0 , provided that $Z<\bar{Z}$, but jumps to infinity when $Z=\bar{Z}^{8}$.

In the following, $\bar{x}$ denotes the flow of non-renewable resource that could be used at the ceiling, without any sequestration scheme, that is, the solution of $\dot{Z}_{t}=\zeta x_{t}-s_{t}-\alpha Z_{t}$ for $s_{t}=0$ and $Z_{t}=\bar{Z}$, hence $\bar{x}=\alpha \bar{Z} / \zeta$. We use $\bar{p}_{x}$ to denote the corresponding price, $\bar{p}_{x}=u^{\prime}(\bar{x})$. Clearly, if $\bar{p}_{x}$ were lower than $c_{x}$, there would be no ceiling problem since, even if the resource rent would be nil, the optimal consumption of the polluting resource would remain below $\bar{x}$. Thus, we assume:

Assumption A.2 : $\bar{p}_{x}>c_{x}$, which is equivalent to $\tilde{x}>\bar{x}$.

However, if sequestration needs to be used, we must consider an even stronger assumption: the total marginal cost of a clean consumption of coal, $c_{x}+c_{s} \zeta$, must be lower than $\bar{p}_{x}$. If not, it would always be better to stay constrained at $\bar{x}$ rather than relaxing the constraint by sequestering some part of the emission flow.

Assumption A.3: $\bar{p}_{x}>c_{x}+c_{s} \zeta$.

\footnotetext{
${ }^{7}$ It is essential for the results that the natural regeneration flow should be some increasing function $f$ of the pollution stock. The specification $f(Z)=\alpha Z$ is assumed for the sake of analytical tractability. For a discussion of the problems raised by non-increasing functions, see Tahvonen and Salo (1996), Tahvonen and Withagen (1996) and Toman and Withagen (1996).

${ }^{8}$ For standard results on optimal mining with a smooth damage function, see Tahvonen (1997).
} 
As discussed in section 4 (subsection 4.1), Assumption A.3 is a necessary but not a sufficient condition.

The other resource is a renewable resource that can be made available to the end users at a constant average cost $c_{y}$ (hence the same constant marginal cost). The cost of the renewable resource is the total cost of supplying the good to the final users, so that the non-renewable and the renewable resources are perfect substitutes for the users. Let us assume that $\bar{y}$ is the constant instantaneous flow of renewable resource available at each point of time, and that this resource is non-storable in the long term, except at a prohibitive storage cost. Let $y_{t}$ be the part of the available flow consumed at time $t$, so the part $\bar{y}-y_{t}$ of the flow that is not immediately consumed is definitely lost.

Concerning the monetary costs alone, i.e. those not involving any scarcity rents, we assume that the cost of the non-renewable resource is lower than the cost of the renewable resource. In the present case, this corresponds to the main renewable energies ${ }^{9}$ and the main non-renewable energies.

Assumption A.4 : $c_{x}<c_{y}$.

Let $\tilde{y}$ be the flow of renewable resource society would have to consume once the nonrenewable resource is exhausted, provided that $\bar{y}$ is sufficiently large. $\tilde{y}$ is the solution of $u^{\prime}(y)=c_{y}: \tilde{y}=d\left(c_{y}\right)$. Chakravorty et al. (2004) showed that, for $\bar{y}<\tilde{y}$ and without any sequestration opportunity, we may have many different optimal paths since a rent has to be charged for the use of the renewable resource even before the exhaustion of the non-renewable resource. For the sake of simplicity, we first assume in sections 3 and 4 that the renewable resource is abundant. By abundant, we mean that, at the marginal cost $c_{y}$, the quantity to be supplied is, at the very most, equal to $\bar{y}$. We also assume that $c_{y}>\bar{p}_{x}$ so that, when the coal consumption is bounded at the ceiling, the renewable resource is not competitive.

Assumption A.5 : $\bar{y}>\tilde{y}$ and $c_{y}>\bar{p}_{x}$.

Under A.4 and A.5, the phase of active sequestration always precedes the phase of renewable resource use as shown in section 4. To obtain a phase of active sequestration

\footnotetext{
${ }^{9}$ An important exception is hydroelectricity.
} 
combined with the use of renewable energy, we must assume not only that $\bar{y}<\tilde{y}$, but also that $c_{y}<\bar{p}_{x y}$ where $\bar{p}_{x y}=u^{\prime}(\bar{x}+\bar{y})<\bar{p}_{x}$. Hence, when the stock of pollution is at the ceiling, the renewable energy is competitive since $c_{y}<\bar{p}_{x y}$, and, moreover, both resources have to be used. In this case, the non-renewable resource is limited at $\bar{x}$ by the pollution stock constraint and the renewable resource is constrained at $\bar{y}$ by the available supply. Thus, by using $\bar{p}_{y}$ to denote the marginal gross surplus at $\bar{y}$, we can assume alternatively:

Assumption A.6 $: \bar{y}<\tilde{y}$ and $\bar{y}<\bar{x}$, or equivalently $\bar{p}_{x y}>c_{y}$ and $\bar{p}_{y}>\bar{p}_{x y}$. Furthermore, $\bar{p}_{x y}>c_{x}+c_{s} \zeta$.

Under A.6, we must add another constraint: the renewable energy consumption cannot be higher than $\bar{y}$ :

$$
\bar{y}-y_{t} \geq 0
$$

Let us assume that the instantaneous social rate of discount, $\rho>0$, is constant and the objective of the social planner is to choose the resource and abatement trajectories that maximize the sum of the discounted instantaneous net surplus.

\subsection{Problem formulation}

The social planner problem can be expressed as follows $(P)$ :

$$
\max _{\left\{\left(s_{t}, x_{t}, y_{t}\right), t \geq 0\right\}} \int_{0}^{\infty}\left[u\left(x_{t}+y_{t}\right)-c_{s} s_{t}-c_{x} x_{t}-c_{y} y_{t}\right] e^{-\rho t} d t
$$

s.t. (1) to (6), $X_{0}=X^{0}, Z_{0}=Z^{0}<\bar{Z}, S^{0}=0, s_{t} \geq 0, x_{t} \geq 0$ and $y_{t} \geq 0$.

Let $\mathcal{L}$ be the current value Lagrangian for the problem $(P)$ :

$$
\begin{aligned}
\mathcal{L}= & u\left(x_{t}+y_{t}\right)-c_{s} s_{t}-c_{x} x_{t}-c_{y} y_{t}-\lambda_{t} x_{t}+\eta_{t} s_{t}+\nu_{S t}\left[\bar{S}-S_{t}\right] \\
& +\mu_{t}\left[\zeta x_{t}-s_{t}-\alpha Z_{t}\right]+\nu_{Z t}\left[\bar{Z}-Z_{t}\right]+\bar{\gamma}_{s t}\left[\zeta x_{t}-s_{t}\right]+\gamma_{s t} s_{t} \\
& +\gamma_{x t} x_{t}+\bar{\gamma}_{y t}\left[\bar{y}-y_{t}\right]+\gamma_{y t} y_{t} .
\end{aligned}
$$


The first-order conditions (FOCs) and complementary slackness conditions are:

$$
\begin{aligned}
\partial \mathcal{L} / \partial s_{t}=0 \Leftrightarrow & c_{s}=\eta_{t}-\mu_{t}-\bar{\gamma}_{s t}+\gamma_{s t} \\
\partial \mathcal{L} / \partial x_{t}=0 \Leftrightarrow & u^{\prime}\left(x_{t}+y_{t}\right)=c_{x}+\lambda_{t}-\mu_{t} \zeta-\bar{\gamma}_{s t} \zeta-\gamma_{x t} \\
\partial \mathcal{L} / \partial y_{t}=0 \Leftrightarrow & u^{\prime}\left(x_{t}+y_{t}\right)=c_{y}+\bar{\gamma}_{y t}-\gamma_{y t} \\
& \bar{\gamma}_{s t} \geq 0 \quad \text { and } \quad \bar{\gamma}_{s t}\left[\zeta x_{t}-s_{t}\right]=0 \\
& \gamma_{s t} \geq 0 \quad \text { and } \quad \gamma_{s t} s_{t}=0 \\
& \gamma_{x t} \geq 0 \quad \text { and } \quad \gamma_{x t} x_{t}=0 \\
& \bar{\gamma}_{y t} \geq 0 \text { and } \quad \bar{\gamma}_{y t}\left[\bar{y}-y_{t}\right]=0 \\
& \gamma_{y t} \geq 0 \text { and } \quad \gamma_{y t} y_{t}=0 .
\end{aligned}
$$

Note that, under the assumption A.1, A.4 and A.5, the condition (9) implies that $x_{t}+y_{t}$ is at least equal to $\tilde{y}$ and that $y_{t}$ is at most equal to $\tilde{y}<\bar{y}^{10}$. Thus, $\bar{\gamma}_{y t}$ must be equal to $0, t \geq 0$.

The dynamics of the costate variables must satisfy:

$$
\begin{aligned}
\dot{\lambda}_{t}=\rho \lambda_{t}-\partial \mathcal{L} / \partial X & \Leftrightarrow \quad \dot{\lambda}_{t}=\rho \lambda_{t} \\
\dot{\eta}_{t}=\rho \eta_{t}-\partial \mathcal{L} / \partial S & \Leftrightarrow \quad \dot{\eta}_{t}=\rho \eta_{t}+\nu_{S t} \\
\dot{\mu}_{t}=\rho \mu_{t}-\partial \mathcal{L} / \partial Z & \Leftrightarrow \quad \dot{\mu}_{t}=(\alpha+\rho) \mu_{t}+\nu_{Z t}
\end{aligned}
$$

with the following associated complementary slackness conditions:

$$
\begin{array}{lll}
\nu_{S t} \geq 0 & \text { and } & \nu_{S t}\left[\bar{S}-S_{t}\right]=0 \\
\nu_{Z t} \geq 0 & \text { and } & \nu_{Z t}\left[\bar{Z}-Z_{t}\right]=0 .
\end{array}
$$

Note that (15) implies that $\lambda_{t}=\lambda_{0} e^{\rho t}$. Hence, the transversality condition for $X_{t}$ takes the following form:

$$
\lim _{t \uparrow \infty} e^{-\rho t} \lambda_{t} X_{t}=\lambda_{0} \lim _{t \uparrow \infty} X_{t}=0 .
$$

The other transversality conditions are:

$$
\begin{aligned}
& \lim _{t \uparrow \infty} e^{-\rho t} \eta_{t} S_{t}=0 \\
& \lim _{t \uparrow \infty} e^{-\rho t} \mu_{t} Z_{t}=0 .
\end{aligned}
$$

\footnotetext{
${ }^{10}$ If $y_{t}>0$ then $\gamma_{y t}=0$, thus $u^{\prime}\left(x_{t}+y_{t}\right)=c_{y}+\bar{\gamma}_{y t}$. Since $u^{\prime}$ is decreasing, the highest value of $y_{t}$ solution of (9) is obtained for $x_{t}=0$ and for $\bar{\gamma}_{y t}=0$, which is possible under the abundance assumption A.5. Thus, in this case, $y_{t}$ is precisely equal to $\tilde{y}$.
} 
Clearly the costate variables $\eta_{t}$ and $\mu_{t}$ are non-positive. Furthermore, given that $S_{t}$ is non-decreasing and starting from $S_{0}=S^{0}=0$, there must exist some time interval $[0, \bar{t})$ during which $S_{t}<\bar{S}$, hence $\nu_{S t}=0$, so that integrating (16) we get:

$$
\eta_{t}=\eta_{0} e^{\rho t} \quad, t \in[0, \bar{t}) .
$$

By the same type of argument, we get for any time interval $\left[t_{0}, t_{1}\right)$ during which $Z_{t}<\bar{Z}$, we obtain:

$$
\mu_{t}=\mu_{t_{0}} e^{(\alpha+\rho)\left(t-t_{0}\right)} \quad, t \in\left[t_{0}, t_{1}\right)
$$

Since $Z^{0}<\bar{Z}$, there must exist some initial interval with $t_{0}=0$ and $t_{1}>0$, so that $\mu_{t}=\mu_{0} e^{(\alpha+\rho) t}, t \in\left[0, t_{1}\right)$. Note also that, since $X^{0}$ is finite, there must be some time $t_{2}$ from which $Z_{t}<\bar{Z}, t \geq t_{2}$, so that $\mu_{t}=0, t \geq t_{2}$.

\section{$3 \quad$ Hotelling and optimal paths without any carbon sink}

\subsection{Pure Hotelling paths}

Without any ceiling constraint and under A.5, the FOCs (8) and (9) would be:

$$
\begin{aligned}
& u^{\prime}\left(x_{t}+y_{t}\right)=c_{x}+\lambda_{0} e^{\rho t}-\gamma_{x t} \\
& u^{\prime}\left(x_{t}+y_{t}\right)=c_{y}-\gamma_{y t},
\end{aligned}
$$

together with the complementary slackness conditions (12)-(14). Thus, if both $x_{t}$ and $y_{t}$ were strictly positive over some non-degenerated time interval, we would have $u^{\prime}\left(x_{t}+y_{t}\right)=$ $c_{x}+\lambda_{0} e^{\rho t}=c_{y}$ over the interval, which is clearly impossible. Hence, the resources have to be exploited sequentially; first, the less costly one, i.e. the non-renewable resource, and next, the more costly one, i.e. the renewable resource. Moreover, the initial value of the coal rent $\lambda_{0}$, is at most equal to $c_{y}-c_{x}$.

For any $\lambda_{0} \in\left(0, c_{y}-c_{x}\right)$, let $t^{H}\left(\lambda_{0}\right)$ be that time at which $c_{x}+\lambda_{0} e^{\rho t}=c_{y}$, and let $d_{t}^{H}\left(\lambda_{0}\right)=d\left(c_{x}+\lambda_{0} e^{\rho t}\right), t \in\left[0, t^{H}\left(\lambda_{0}\right)\right)$. The optimal value of $\lambda_{0}, \lambda_{0}^{H}$, is given as the unique solution of the cumulative demand-initial endowment balance equation:

$$
\int_{0}^{t^{H}\left(\lambda_{0}\right)} d_{t}\left(\lambda_{0}\right) d t=X^{0}
$$

The optimal consumption would then be the standard Hotelling solution:

$$
x_{t}=\left\{\begin{array}{ll}
d_{t}^{H}\left(\lambda_{0}^{H}\right) & , t<t^{H}\left(\lambda_{0}^{H}\right) \\
0 & , t^{H}\left(\lambda_{0}^{H}\right) \leq t
\end{array} \quad \text { and } \quad y_{t}=\left\{\begin{array}{ll}
0 & , t<t^{H}\left(\lambda_{0}^{H}\right) \\
\tilde{y} & , t^{H}\left(\lambda_{0}^{H}\right) \leq t
\end{array} .\right.\right.
$$


All the optimality conditions are satisfied by the following values of $\gamma_{x t}$ and $\gamma_{y t}$ :

$\gamma_{x t}=\left\{\begin{array}{ll}0 & , t<t^{H}\left(\lambda_{0}^{H}\right) \\ c_{x}+\lambda_{0} e^{\rho t}-c_{y} & , t^{H}\left(\lambda_{0}^{H}\right) \leq t\end{array} \quad\right.$ and $\quad \gamma_{y t}=\left\{\begin{array}{ll}c_{y}-c_{x}-\lambda_{0} e^{\rho t} & , t<t^{H}\left(\lambda_{0}^{H}\right) \\ 0 & , t^{H}\left(\lambda_{0}^{H}\right) \leq t\end{array}\right.$.

As a function of $X^{0}, \lambda_{0}^{H}$ strictly decreases with ${ }^{11}$ :

$$
\lim _{X^{0} \downarrow 0} \lambda_{0}^{H}=c_{y}-c_{x} \quad \text { and } \quad \lim _{X^{0} \uparrow \infty} \lambda_{0}^{H}=0 .
$$

Let $Z_{t}^{H}\left(\lambda_{0}\right)$ be the trajectory of the carbon stock generated by the coal consumption flow $d_{t}^{H}\left(\lambda_{0}\right) . Z_{t}^{H}\left(\lambda_{0}\right)$ is the solution of:

$$
\dot{Z}_{t}=\zeta d_{t}^{H}\left(\lambda_{0}\right)-\alpha Z_{t}, t \in\left[0, t^{H}\left(\lambda_{0}\right)\right)
$$

together with the initial condition $Z_{0}=Z^{0}$. Here, we define $Z_{m}^{H}\left(\lambda_{0}\right)$ as the maximum quantity of atmospheric carbon over the interval $\left[0, t^{H}\left(\lambda_{0}\right)\right)^{12}$ :

$$
Z_{m}^{H}\left(\lambda_{0}\right)=\sup \left\{Z_{t}^{H}\left(\lambda_{0}\right), t \in\left[0, t^{H}\left(\lambda_{0}\right)\right)\right\}
$$

Under A.2, when $\lambda_{0}$ is sufficiently low (that is, $X^{0}$ is sufficiently high), then $Z_{m}^{H}\left(\lambda_{0}\right)>$ $\bar{Z}^{13}$. Let $\bar{X}_{0}$ be the value of $X_{0}$ for which $Z_{m}^{H}\left(\lambda_{0}\left(X^{0}\right)\right)=\bar{Z}$. Taking into account the ceiling constraint, it can be easily seen that, for $X^{0}$ lower than $\bar{X}_{0}$, the constraint would never be tight and the optimal consumption path would be above the standard Hotelling path as given by (27). In the following, we assume that the ceiling constraint would be violated along the pure Hotelling path.

Assumption A.7 : $X^{0}>\bar{X}^{0}$.

\subsection{Optimal paths with no active abatement opportunity}

Let us assume that there is no abatement opportunity apart from the natural regeneration process. It has been shown by Chakravorty et al. (2004) that, under A.1, A.2, A.4 and A.5, the optimal consumption path is a four phases path as illustrated in Figure 1 below.

\footnotetext{
${ }^{11}$ Firstly, $t^{H}\left(\lambda_{0}\right)$ is a strictly decreasing function of $\lambda_{0}$ with $\lim _{\lambda_{0} \downarrow 0} t^{H}=\infty$ and $\lim _{\lambda_{0} \uparrow\left(c_{y}-c_{x}\right)} t^{H}=0$, and secondly, for any $t \geq 0, \lim _{\lambda_{0} \downarrow 0} d_{t}^{H}=\tilde{x}$ and $\lim _{\lambda_{0} \uparrow\left(c_{y}-c_{x}\right)} d_{t}^{H}=0$.

${ }^{12}$ After $t^{H}\left(\lambda_{0}\right)$, the use of coal is nil, hence $Z_{t}$ is decreasing.

${ }^{13}$ For $\lambda_{0}=0, d_{t}^{H}(0)=\tilde{x}, t \geq 0$, hence $\dot{Z}_{t}=\zeta \tilde{x}-\alpha Z_{t}, Z_{0}=Z^{0}$, yielding the solution $Z_{t}=\zeta \tilde{x} / \alpha+$ $\left(Z^{0}-\zeta \tilde{x} / \alpha\right) e^{-\alpha t}$, so that $\lim _{t \uparrow \infty} Z_{t} \equiv \tilde{Z}=\zeta \tilde{x} / \alpha$. Under A.2, $\tilde{x}>\bar{x}$, hence $\tilde{Z}>\bar{Z}$.
} 
During the first phase $\left[0, t_{1}\right)$, the constraint is slack and only coal has to be used: $q_{t}=x_{t}=d\left(c_{x}+\lambda_{0} e^{\rho t}-\mu_{0} e^{(\alpha+\rho) t} \zeta\right)$, with $\lambda_{0}$ and $\left|\mu_{0}\right|$ sufficiently low so that $x_{t}>\bar{x}$. Since $x_{t}>\bar{x}$ and $Z_{t}<\bar{Z}$, then $Z_{t}$ is increasing because $\zeta x_{t}>\alpha Z_{t}$ : the emission rate is higher than the natural regeneration flow. At $t_{1}$, the carbon ceiling is reached and the full marginal cost of coal, $c_{x}+\lambda_{0} e^{\rho t}-\mu_{0} e^{(\alpha+\rho) t} \zeta$, is equal to $\bar{p}_{x}$.

The second phase $\left[t_{1}, t_{2}\right)$ occurs at the ceiling, when the coal consumption - the only energy being used - is constrained to $\bar{x}$. Thus, the energy price is constant and equal to $\bar{p}_{x}$. Since $p_{t}=\bar{p}_{x}=c_{x}+\lambda_{0} e^{\rho t}-\mu_{0} e^{(\alpha+\rho) t} \zeta$, then $\left|\mu_{t}\right|$ must be decreasing during this phase. At $t_{2}, \mu_{t}=0$ and the ceiling constraint will no longer be active from $t_{2}$ onwards.

The third phase $\left[t_{2}, t_{3}\right)$ is a pure Hotelling phase during which only coal is used: $p_{t}=$ $c_{x}+\lambda_{0} e^{\rho t}$ and $q_{t}=x_{t}=d\left(p_{t}\right)$. Thus, the coal consumption decreases, as seen during the first phase, and the stock is exhausted at the end of the phase. Then, the price must become equal to the marginal cost of the renewable resource $c_{y}$.

During the last phase $\left[t_{3}, \infty\right)$, only the renewable resource is used, $q_{t}=y_{t}=\tilde{y}$ and the price is constant at $c_{y}$.

Both price and quantity paths are illustrated in Figure 1. The hatched surface under the $x_{t}$ curve must be equal to $X^{0}$.

Figure 1 here

We need to determine the optimal values $\lambda_{0}^{n s}, \mu_{0}^{n s}, t_{1}^{n s}, t_{2}^{n s}$ and $t_{3}^{n s}$ ( $n s$ stands for no sequestration, which is forced here) of the five fundamental variables $\lambda_{0}, \mu_{0}, t_{1}, t_{2}$ and $t_{3}$ that solve the following system of five equations:

- The cumulative coal consumption-initial stock balance equation:

$$
\int_{0}^{t_{1}} d\left(c_{x}+\lambda_{0} e^{\rho t}-\mu_{0} e^{(\alpha+\rho) t} \zeta\right) d t+\left[t_{2}-t_{1}\right] \bar{x}+\int_{t_{2}}^{t_{3}} d\left(c_{x}+\lambda_{0} e^{\rho t}\right) d t=X^{0} .
$$

- The price continuity equation at $t_{1}$ :

$$
c_{x}+\lambda_{0} e^{\rho t_{1}}-\mu_{0} e^{(\alpha+\rho) t_{1}} \zeta=\bar{p}_{x} .
$$


- The pollution stock continuity equation at $t_{1}$ :

$$
Z_{t_{1}}\left(\lambda_{0}, \mu_{0}\right)=\bar{Z}
$$

where $Z_{t}\left(\lambda_{0}, \mu_{0}\right)$ is the solution of:

$$
\dot{Z}_{t}=\zeta d\left(c_{x}+\lambda_{0} e^{\rho t}-\mu_{0} e^{(\alpha+\rho) t} \zeta\right)-\alpha Z_{t}, Z_{0}=Z^{0}
$$

- The price continuity equations at $t_{2}$ and $t_{3}$ :

$$
c_{x}+\lambda_{0} e^{\rho t_{2}}=\bar{p}_{x} \quad \text { and } \quad c_{x}+\lambda_{0} e^{\rho t_{3}}=c_{y} .
$$

Chakravorty et al. (2004) demonstrated that, solving the above system of equations for for $\lambda_{0}^{n s}, \mu_{0}^{n s}, t_{1}^{n s}, t_{2}^{n s}$ and $t_{3}^{n s}$ provides values of the other multipliers that satisfy all the optimality conditions.

\section{The case of an abundant renewable substitute}

Although the coal consumption is constrained over a certain time interval $\left[t_{1}, t_{2}\right)$ under assumption A.6, as shown in subsection 3.2 and illustrated in Figure 1, it is not clear a priori whether it is worth relaxing this constraint by sequestering the carbon because sequestration is costly. We first show that there is a very simple test of the optimality of sequestration for relaxing the ceiling constraint. Assuming that it is optimal to sequester, we next determine the optimal policy according to whether the sink or reservoir capacity $\bar{S}$ is large (in subsection 4.2) or small (in subsection 4.3). Large and small capacities are endogenous characteristics of the sink that depend upon all the other fundamentals of the model.

\subsection{Testing the optimality of the sequestration opportunity}

Let us consider the optimal paths determined in subsection 3.2 under a forced condition of no sequestration, and assume two hypothetical values $c_{s}^{\prime}$ and $c_{s}^{\prime \prime}$ (where $c_{s}^{\prime}<c_{s}^{\prime \prime}$ ) of the marginal cost of sequestration. We assume $c_{s}^{\prime}$ is so low at $t_{1}^{n s}$ that $c_{x}+\lambda_{0}^{n s} e^{\rho t}+c_{s}^{\prime} \zeta$ is lower than $\bar{p}_{x}$, while $c_{s}^{\prime \prime}$ is so high at $t_{1}^{n s}$ that $c_{x}+\lambda_{0}^{n s} e^{\rho t}+c_{s}^{\prime \prime} \zeta$ is higher than $\bar{p}_{x}$, as illustrated in Figure 1. 
In the first case, where $c_{x}+\lambda_{0}^{n s} e^{\rho t_{1}^{n s}}+c_{s}^{\prime} \zeta<\bar{p}_{x}$, there is a certain time interval $\left[t_{1}^{n s}, t_{1}^{n s}+\delta\left(c_{s}^{\prime}\right)\right), \delta\left(c_{s}^{\prime}\right)>0$, during which $c_{x}+\lambda_{0}^{n s} e^{\rho t}+c_{s}^{\prime} \zeta$ is lower than $\bar{p}_{x}$ while $Z_{t}=\bar{Z}$. Thus, over this interval, the instantaneous marginal gross surplus generated by $\bar{x}$, that is $\bar{p}_{x}$, is higher than the full marginal cost of supplying a "clean" coal to the final users (that is $\left.c_{x}+\lambda_{0}^{n s} e^{\rho t}+c_{s}^{\prime} \zeta\right)$ provided that the shadow cost charged to the use of the sink is nil. Whatever the capacity of the sink, this capacity will not be saturated if the sequestration rate is sufficiently low. Hence, slightly augmenting the coal consumption within the interval would still allow the net social welfare to increase even if coal consumption would have to be reduced later. For example, increasing the coal consumption by $d x_{t}>0$ at time $t$ within the interval and decreasing it by the same amount at some date $t^{\prime}$ within the interval $\left(t_{2}^{n s}, t_{3}^{n s}\right)$, results in a net benefit equal to $\left[\left(\bar{p}_{x}-\left(c_{x}+c_{s}^{\prime} \zeta\right)\right) e^{-\rho t}-\lambda_{0}^{n s}\right] d x_{t}>0$ (in value at time 0 ).

In the second case, the marginal cost of clean coal consumption, $c_{x}+\lambda_{0}^{n s} e^{\rho t}+c_{s}^{\prime \prime} \zeta$, is always higher than the marginal gross surplus of the energy consumption. Thus, resorting to a sequestration scheme cannot increase the optimized value of the objective function of problem $(P)$.

Clearly, there exists some critical value of the sequestration marginal cost, denoted by $\bar{c}_{s}$, below which relaxation of the ceiling constraint must be used, and above which it must be abandoned. This threshold value is the solution of $c_{x}+\lambda_{0}^{n s} e^{\rho t_{1}^{n s}}+c_{s} \zeta=\bar{p}_{x}$, that is:

$$
\bar{c}_{s}=\left[\bar{p}_{x}-\left(c_{x}+\lambda_{0}^{n s} e^{\rho t_{1}^{n s}}\right)\right] / \zeta
$$

Assumption A.8 : $c_{s}<\bar{c}_{s}$.

In the following, we assume that A.8 applies.

\subsection{The large reservoir case}

In this case, even if no rent is charged for the use of the sink capacity, the reservoir capacity constraint $\bar{S}-S_{t}$ is never active, hence $\eta_{t}=0, t \geq 0$. We note that for $\eta_{t}=0$ and for $s_{t}>0$ implying that $\gamma_{s t}=0$, then the optimality condition (7) becomes:

$$
-\mu_{t}=c_{s}+\bar{\gamma}_{s t}
$$


Next, substituting the above value of $-\mu_{t}$ into the optimality condition (8), bearing in mind $s_{t}>0$ implies that $x_{t}>0$, hence $\gamma_{x t}=0$. We thus obtain:

$$
u^{\prime}\left(x_{t}\right)=c_{x}+c_{s} \zeta+\lambda_{0} e^{\rho t}
$$

which, in turn, implies that:

$$
x_{t}=d\left(c_{x}+c_{s} \zeta+\lambda_{0} e^{\rho t}\right)
$$

However, only part of the emission flow, represented by $\zeta\left[d\left(c_{x}+c_{s} \zeta+\lambda_{0} e^{\rho t}\right)-\bar{x}\right]$, has to be sequestrated. When the ceiling constraint is binding, the instantaneous marginal full cost of a clean unit of coal is denoted by $c_{m}$ :

$$
c_{m}=\left\{\begin{array}{ll}
c_{x}+\lambda_{0} e^{\rho t} & , x_{t}<\bar{x} \\
c_{x}+\lambda_{0} e^{\rho t}+c_{s} \zeta & , x_{t}>\bar{x}
\end{array} .\right.
$$

For $x<\bar{x}$, the regeneration rate $\alpha \bar{Z}$ is higher than the emission rate, whereas the opposite holds for $x>\bar{x}$ so that society has to sequestrate the emission at the margin.

The determination of $x_{t}$ during the sequestration phase is illustrated in Figure 2. Note that, as time passes, $c_{x}+\lambda_{0} e^{\rho t}$ and $c_{x}+c_{s} \zeta+\lambda_{0} e^{\rho t}$ are shifted vertically and upwards. Hence, the sequestration phase is necessarily followed by a phase during which $c_{x}+\lambda_{0} e^{\rho t}<$ $u^{\prime}(\bar{x})<c_{x}+c_{s} \zeta+\lambda_{0} e^{\rho t}$ so that it becomes optimal to consume $\bar{x}$. Although the ceiling is constraining the coal consumption, it is no longer optimal to sequester carbon emissions. This phase at the ceiling is itself followed by a pure Hotelling phase during which $x_{t}<\bar{x}$, once $t$ is sufficiently high so that the $u^{\prime}(x)$ curve intersects the horizontal line $c_{x}+\lambda_{0} e^{\rho t}$ before $\bar{x}$.

Figure 2 here

Thus, the optimal path consists of five phases as illustrated in Figure 3.

Figure 3 here

During the first phase $\left[0, t_{1}\right)$, which takes place below the ceiling, $p_{t}=c_{x}+\lambda_{0} e^{\rho t}-$ $\mu_{0} e^{(\alpha+\rho) t} \zeta<c_{x}+\lambda_{0} e^{\rho t}+c_{s} \zeta<\bar{p}_{x}$, and $q_{t}=x_{t}=d\left(p_{t}\right)>\bar{x}$, so the pollution stock is increasing. At the end of the phase, $p_{t}=c_{x}+\lambda_{0} e^{\rho t}+c_{s} \zeta$ and the ceiling is attained. 
The second phase $\left[t_{1}, t_{2}\right)$ is a phase at the ceiling: $p_{t}=c_{x}+\lambda_{0} e^{\rho t}+c_{s} \zeta<\bar{p}_{x}$ and $q_{t}=x_{t}=d\left(p_{t}\right)>\bar{x}$. A part $\zeta\left[d\left(p_{t}\right)-\bar{x}\right]$ of the potential emission flow is sequestered so that the pollution flow is equal to $\zeta \bar{x}$. At the end of the phase, $p_{t}=\bar{p}_{x}$.

The third phase $\left[t_{2}, t_{3}\right)$ is at the ceiling, but without sequestration: $p_{t}=\bar{p}_{x}$ and $q_{t}=x_{t}=\bar{x}$, during which $\left|\mu_{t}\right|$ is decreasing. At the end of the phase, $\mu_{t}=0$.

The fourth phase $\left[t_{3}, t_{4}\right)$ is a pure Hotelling phase: $p_{t}=c_{x}+\lambda_{0} e^{\rho t}$ and $q_{t}=x_{t}=d\left(p_{t}\right)$. At the end of this phase, the price of energy is just equal to the marginal cost of the renewable resource $c_{y}$ and the coal is exhausted.

The last phase $\left[t_{4}, \infty\right)$ is a phase during which the only renewable resource is used.

We now need to determine the values of the six variables $\lambda_{0}, \mu_{0}, t_{1}, t_{2}, t_{3}$ and $t_{4}$. They are provided by solving the following system of six equations:

- The cumulative demand-supply balance equation, which is written here as:

$$
\begin{aligned}
& \int_{0}^{t_{1}} d\left(c_{x}+\lambda_{0} e^{\rho t}-\mu_{0} e^{(\alpha+\rho) t} \zeta\right) d t+\int_{t_{1}}^{t_{2}} d\left(c_{x}+\lambda_{0} e^{\rho t}+c_{s} \zeta\right) d t \\
&+\left[t_{3}-t_{2}\right] \bar{x}+\int_{t_{3}}^{t_{4}} d\left(c_{x}+\lambda_{0} e^{\rho t}\right) d t=X^{0} .
\end{aligned}
$$

- The price continuity equation at $t_{1}$ :

$$
c_{x}+\lambda_{0} e^{\rho t_{1}}-\mu_{0} e^{(\alpha+\rho) t_{1}} \zeta=c_{x}+\lambda_{0} e^{\rho t_{1}}+c_{s} \zeta
$$

- The pollution stock continuity equation at $t_{1}$ :

$$
Z_{t_{1}}\left(\lambda_{0}, \mu_{0}\right)=\bar{Z}
$$

- The price continuity equations at $t_{2}, t_{3}$ and $t_{4}$ :

$$
\begin{aligned}
& c_{x}+\lambda_{0} e^{\rho t_{2}}+c_{s} \zeta=\bar{p}_{x} \\
& c_{x}+\lambda_{0} e^{\rho t_{3}}=\bar{p}_{x} \\
& c_{x}+\lambda_{0} e^{\rho t_{4}}=c_{y} .
\end{aligned}
$$

Let $\lambda_{0}^{l r}, \mu_{0}^{l r}$ and $t_{1}^{l r}$ to $t_{4}^{l r}$ (lr stands for large reservoir) represent the values obtained by solving the above system of equations. In the Appendix, we show that, for these values of $\lambda_{0}, \mu_{0}, t_{1}$ to $t_{4}$, the other multipliers have values satisfying all the optimality conditions. 
We can now give a precise definition of a large reservoir. A reservoir or a sink is said to be large if it allows for the carbon to be effectively sequestered as prescribed by the above policy, that is:

$$
\bar{S} \geq \int_{t_{1}^{l r}}^{t_{2}^{l r}}\left[d\left(c_{x}+\lambda_{0}^{l r} e^{\rho t}+c_{s} \zeta\right)-\bar{x}\right] d t .
$$

The reservoir will be said small if such a carbon mass cannot be sequestered.

\subsection{The small reservoir case}

If the reservoir is small, its shadow cost $\eta_{t}$ cannot be nil. We know that, as long as the reservoir is not saturated, the absolute value of $\eta_{t}$ is increasing at the social rate of discount $^{14}: \eta_{t}=\eta_{0} e^{\rho t}$. Thus, the full marginal cost of clean coal is given by $c_{x}+\lambda_{0} e^{\rho t}+$ $\left(c_{s}-\eta_{0} e^{\rho t}\right) \zeta$.

As in the case of a large reservoir, the optimal path consists of five phases. The only difference is that $p_{t}=c_{x}+\lambda_{0} e^{\rho t}+\left(c_{s}-\eta_{0} e^{\rho t}\right) \zeta$ during the second phase $\left[t_{1}, t_{2}\right)$, when it is optimal to sequester part of the potential emission flow represented by $\zeta\left[d\left(p_{t}\right)-\bar{x}\right]$. Also at $t_{2}$, the carbon reservoir capacity $\bar{S}$ must be saturated, i.e. $S_{t_{2}}=\bar{S}$ and $S_{t}<\bar{S}, t<t_{2}$.

We now have to determine the values of the seven variables $\lambda_{0}, \mu_{0}, \eta_{0}$ and $t_{1}$ to $t_{4}$. They are obtained by solving the system of seven equations below:

- The cumulative demand-supply balance equations:

$$
\begin{array}{r}
\int_{0}^{t_{1}} d\left(c_{x}+\lambda_{0} e^{\rho t}-\mu_{0} e^{(\alpha+\rho) t} \zeta\right) d t+\int_{t_{1}}^{t_{2}} d\left(c_{x}+\lambda_{0} e^{\rho t}+\left(c_{s}-\eta_{0} e^{\rho t}\right) \zeta\right) d t \\
+\left[t_{3}-t_{2}\right] \bar{x}+\int_{t_{3}}^{t_{4}} d\left(c_{x}+\lambda_{0} e^{\rho t}\right) d t=X^{0}
\end{array}
$$

- The price continuity equation at $t_{1}$ :

$$
c_{x}+\lambda_{0} e^{\rho t_{1}}-\mu_{0} e^{(\alpha+\rho) t_{1}} \zeta=c_{x}+\lambda_{0} e^{\rho t_{1}}+\left(c_{s}-\eta_{0} e^{\rho t_{1}}\right) \zeta
$$

- The pollution stock continuity equation at $t_{1}$ :

$$
Z_{t_{1}}\left(\lambda_{0}, \mu_{0}\right)=\bar{Z}
$$

This equation is the same as in the previous case of a large reservoir.

\footnotetext{
${ }^{14}$ Remember that $\eta_{t}<0$.
} 
- The price continuity equation at $t_{2}$ :

$$
c_{x}+\lambda_{0} e^{\rho t_{2}}+\left(c_{s}-\eta_{0} e^{\rho t_{2}}\right) \zeta=\bar{p}_{x} .
$$

- The reservoir capacity saturation equation at $t_{2}$ :

$$
\zeta \int_{t_{1}}^{t_{2}}\left[d\left(c_{x}+\lambda_{0} e^{\rho t}+\left(c_{s}-\eta_{0} e^{\rho t}\right) \zeta\right)-\bar{x}\right] d t=\bar{S}
$$

- The price continuity equations at $t_{3}$ and $t_{4}$ :

$$
c_{x}+\lambda_{0} e^{\rho t_{3}}=\bar{p}_{x} \quad \text { and } \quad c_{x}+\lambda_{0} e^{\rho t_{4}}=c_{y},
$$

These continuity equations are the same as in the case of a large reservoir.

As in the previous case, for these values of $\lambda_{0}, \mu_{0}, \eta_{0}$ and $t_{1}$ to $t_{4}$, we show that the other multipliers have values satisfying all the optimality conditions (see Appendix). The main conclusion of the analysis is that, if sequestration needs to be implemented under A.5, it must occur before using the renewable resource. As discussed in the next section, the optimal policy may be different when solar energy - although relatively inexpensive is not abundant.

\section{The case of a rare renewable substitute}

Let us assume that the renewable energy is not abundant and that A.6 is valid. In that case, without any sequestration opportunity, it would be optimal to use the renewable resource at the ceiling. We first show how to modify the optimality test of the sequestration option. Given that the test is positive, indicating it is optimal to sequester, we next show that there are two types of optimal policy, according to whether the sequestration phase should begin before starting use of the renewable clean substitute. Otherwise, the opposite must apply.

\subsection{Testing the optimality of the sequestration opportunity}

To test the optimality of the sequestration opportunity in the present case, we first have to determine the optimal policy in the absence of an opportunity. In the case of a rare renewable substitute, as well as with an abundant substitute, the decision to sequester or not is endogenously determined. 
The first point to be noticed is that, since $\bar{y}<\tilde{y}$, although the renewable resource is competitive at a price $p_{e}>c_{y}$, it cannot supply the entire market, at least for prices not too far from $c_{y}$. To determine the optimal policy, let us define $d_{n}\left(p_{e}\right)$ as the part of the energy needs that has to be supplied by the non-renewable resource. The other part, if any, is represented by $d\left(p_{e}\right)-d_{n}\left(p_{e}\right)$ and has to be supplied by the renewable resource:

$$
d_{n}\left(p_{e}\right)= \begin{cases}d\left(p_{e}\right) & , p_{e}<c_{y} \\ d\left(p_{e}\right)-\bar{y} & , c_{y} \leq p_{e}<\bar{p}_{y} \\ 0 & , \bar{p}_{y} \leq p_{e}\end{cases}
$$

Under A.6, $\bar{p}_{x y}>c_{y}$, which means we can have two types of optimal paths according to the value of $Z^{0}$. Along the first type of path (see Figure 4), which would appear only for sufficiently low values of $Z^{0}$, the initial price $p_{0}$ is lower than $c_{y}$. Hence, the initial period during which $p_{t}=c_{x}+\lambda_{0} e^{\rho t}-\mu_{0} e^{(\alpha+\rho) t}$ and $Z_{t}<\bar{Z}$ can be split into two phases. During the first phase $\left[0, t_{1}\right)$, when $p_{t}<c_{y}$, coal must be the only energy source, and $q_{t}=x_{t}=d_{n}\left(p_{t}\right)=d\left(p_{t}\right)$. On the other hand, during the second phase $\left[t_{1}, t_{2}\right)$, both coal and solar energy must be used, with $x_{t}=d_{n}\left(p_{t}\right)$ and $y_{t}=\bar{y}$. The phase at the ceiling $\left[t_{2}, t_{3}\right)$ begins at $t_{2}$, when $p_{t}=\bar{p}_{x y}$; throughout this phase, $p_{t}=\bar{p}_{x y}$ and $q_{t}=\bar{x}+\bar{y}$, while, at the end, $\mu_{t}=0$. This phase is followed by a pure Hotelling phase $\left[t_{3}, t_{4}\right)$ as far as prices are concerned: $p_{t}=c_{x}+\lambda_{0} e^{\rho t}, x_{t}=d_{n}\left(p_{t}\right)$ and $y_{t}=\bar{y}$. At $t_{4}, p_{t}=\bar{p}_{y}, x_{t}=0$ and coal is exhausted. During the last phase $\left[t_{4}, \infty\right)$, only the renewable resource is available, so that $p_{t}=\bar{p}_{y}$ and $q_{t}=y_{t}=\bar{y}$.

The values of the six endogenous variables characterizing this type of path are determined by solving the following six-equation system.

- The cumulative coal consumption-initial stock balance equation, written here as:

$$
\int_{0}^{t_{2}} d_{n}\left(c_{x}+\lambda_{0} e^{\rho t}-\mu_{0} e^{(\alpha+\rho) t} \zeta\right) d t+\bar{x}\left[t_{3}-t_{2}\right]+\int_{t_{3}}^{t_{4}} d_{n}\left(c_{x}+\lambda_{0} e^{\rho t}\right) d t=X^{0} .
$$

- The pollution stock continuity equation at $t_{2}$ :

$$
Z_{t_{2}}^{n}\left(\lambda_{0}, \mu_{0}\right)=\bar{Z}
$$

where $Z_{t_{2}}^{n}\left(\lambda_{0}, \mu_{0}\right)$ is the solution of the differential equation ${ }^{15}$ :

\footnotetext{
${ }^{15}$ Since $d_{n}\left(p_{e}\right)$ is discontinuous at $p_{e}=c_{y}$, then technically $Z_{t}^{n}$ is obtained by solving first: $\dot{Z}_{t}=$ $\zeta d\left(c_{x}+\lambda_{0} e^{\rho t}-\mu_{0} e^{(\alpha+\rho) t} \zeta\right)-\alpha Z_{t}, Z_{0}=Z^{0}$. Let $Z_{t}^{(1)}$ be the solution of this differential equation and
} 


$$
\dot{Z}_{t}=\zeta d_{n}\left(c_{x}+\lambda_{0} e^{\rho t}-\mu_{0} e^{(\alpha+\rho) t} \zeta\right)-\alpha Z_{t}, \quad Z_{0}=Z^{0}
$$

- The four price-continuity equations:

$$
\begin{aligned}
& c_{x}+\lambda_{0} e^{\rho t_{1}}-\mu_{0} e^{(\alpha+\rho) t_{1}} \zeta=c_{y} \quad, \quad c_{x}+\lambda_{0} e^{\rho t_{2}}-\mu_{0} e^{(\alpha+\rho) t_{2}} \zeta=\bar{p}_{x y} \\
& c_{x}+\lambda_{0} e^{\rho t_{3}}=\bar{p}_{x y} \quad \text { and } \quad c_{x}+\lambda_{0} e^{\rho t_{4}}=\bar{p}_{y} .
\end{aligned}
$$

The corresponding optimal paths are illustrated in Figure 4, where the hatched surface under the curve $x_{t}$ is equal to $X^{0}$.

Figure 4 here

Now, let us assume that $\left\{\left(p_{t}, x_{t}, y_{t}\right), t \geq 0\right\}$ is an optimal path for initial values $X^{0}$ and $Z^{0}$ of the state variables, and $X_{t}$ and $Z_{t}$ represent, respectively, the remaining coal stock and the pollution stock generated by $x_{t}$. We also consider a given date $t^{\prime}>0$ and the problem $(P)$ with initial conditions $X_{t^{\prime}}$ and $Z_{t^{\prime}}$. Let $\left\{\left(p_{t}^{\prime}, x_{t}^{\prime}, y_{t}^{\prime}\right), t \geq 0\right\}$ be the solution of this new problem. Then, this solution corresponds simply to $p_{t}^{\prime}=p_{t^{\prime}+t}, x_{t}^{\prime}=x_{t^{\prime}+t}$ and $y_{t}^{\prime}=y_{t^{\prime}+t}$. Bearing this in mind, we can see that there is a second type of optimal path starting with $p_{0}>c_{y}$, in which the renewable resource has to be used from the beginning. These paths have only four distinct phases, since there is no longer any first phase as defined in the previous five-phase optimal path model. Clearly, the second type of path is optimal under A.6 when $Z^{0}$ is sufficiently high but nevertheless lower than $\bar{Z}$.

Let $\bar{t}_{x y}$ denote the time at which $p_{t}=\bar{p}_{x y}$. Along paths of the first type, $\bar{t}_{x y}=t_{2}$ at the end of the second phase, whereas, along paths of the second type, $\bar{t}_{x y}=t_{1}$, since $\bar{p}_{x y}$ is reached at the end of the first phase $\left[0, t_{1}\right)$. The optimality test of the sequestration option is the same as for an abundant renewable substitute, except that $\bar{p}_{x y}$ must be taken here as the reference price instead of $\bar{p}_{x}$. The corresponding curve $c_{x}+\lambda_{0} e^{\rho t}+c_{s} \zeta$ must be located at $t=\bar{t}_{x y}$, as illustrated in Figure 4 .

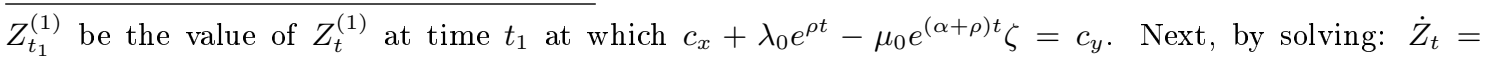
$\zeta\left[d\left(c_{x}+\lambda_{0} e^{\rho t}-\mu_{0} e^{(\alpha+\rho) t} \zeta\right)-\bar{x}\right]-\alpha Z_{t}, Z_{t_{1}}=Z_{t_{1}}^{(1)}$, we obtain $Z_{t_{1}}^{(2)}$ as the solution of this equation. Then, we can write:

$$
Z_{t}^{n}=\left\{\begin{array}{cc}
Z_{t_{1}}^{(1)} & , 0 \leq t \leq t_{1} \\
Z_{t_{1}}^{(2)} & , t_{1} \leq t<t_{2}
\end{array}\right.
$$


The threshold value $\bar{c}_{s}$ represents the average sequestration cost below which it is optimal to sequester and above which it is not. In this case, is here equal to:

$$
\bar{c}_{s}=\left[\bar{p}_{x y}-\left(c_{x}+\lambda_{0}^{n s} e^{\rho \bar{t}_{x y}}\right)\right] / \zeta
$$

where $\lambda_{0}^{n s}$ is the optimal value of $\lambda_{0}$ under a forced no-sequestration policy.

Assumption A.9 $: c_{s}<\bar{c}_{s}$.

In the following, we assume that A.9 is valid.

\subsection{Optimal paths for beginning sequestration before using the renew- able substitute}

Under A.6, when sequestration is not applied, use of the renewable resource must always begin before reaching the ceiling as shown in the preceding section. However, when sequestration has to be used, it may happen that - once at the ceiling - sequestration can be the first scheme required to relax the ceiling constraint. Then, sequestration and the renewable substitute are used jointly, and, finally, only the renewable resource on its own. This case is illustrated in Figure 5, which assumes a small reservoir.

Figure 5 here

In Figure 5, the price path, the demand functions $d\left(p_{e}\right)$ and $d_{n}\left(p_{e}\right)$, and the resource consumption paths are drawn in the North-East, the North-West and the South-East quadrants, respectively. The South-West quadrant is a purely technical device to show how the quantities are derived from the price at the same time.

The optimal path consists of six phases.

The first phase $\left[0, t_{1}\right)$ is the usual phase of coal consumption under the ceiling: $p_{t}=$ $c_{x}+\lambda_{0} e^{\rho t}-\mu_{0} e^{(\alpha+\rho) t} \zeta<c_{x}+\lambda_{0} e^{\rho t}+\left(c_{s}-\eta_{0} e^{\rho t}\right) \zeta$. At the end of this phase, the ceiling is reached and $p_{t_{1}}=c_{x}+\lambda_{0} e^{\rho t_{1}}+\left(c_{s}-\eta_{0} e^{\rho t_{1}}\right) \zeta$.

The second phase $\left[t_{1}, t_{2}\right)$ takes place at the ceiling, during which only coal is consumed, with some part of the potential emissions being sequestered: $p_{t}=c_{x}+\lambda_{0} e^{\rho t}+\left(c_{s}-\eta_{0} e^{\rho t}\right) \zeta$, 
$x_{t}=d_{n}\left(p_{t}\right)=d\left(p_{t}\right)$ and $s_{t}=\left[d_{n}\left(p_{t}\right)-\bar{x}\right] / \zeta$. At the end of this phase, $p_{t_{2}}=c_{y}$ and the renewable energy becomes competitive.

During the third phase $\left[t_{2}, t_{3}\right)$, the constraint is relaxed by the joint use of sequestration and solar energy consumption: $p_{t}=c_{x}+\lambda_{0} e^{\rho t}+\left(c_{s}-\eta_{0} e^{\rho t}\right) \zeta$, as in the previous phase. But because some part of the energy demand is satisfied by the solar energy, the proportion of the emission flow that has to be sequestered is lower: $x_{t}=d_{n}\left(p_{t}\right)=d\left(p_{t}\right)-\bar{x}, s_{t}=$ $\left[d_{n}\left(p_{t}\right)-\bar{x}\right] / \zeta$ and $y_{t}=\bar{y}$. At the end of the phase, $p_{t_{3}}=\bar{p}_{x y}$ and the capacity of the sink is saturated, $S_{t_{3}}=\bar{S}$, so sequestration is no longer of any help.

The fourth phase $\left[t_{3}, t_{4}\right)$ is at the ceiling with both coal and solar energy, but without sequestration: $p_{t}=\bar{p}_{x y}, x_{t}=\bar{x}$ and $y_{t}=\bar{y}$. At the end of this phase, $\mu_{t_{4}}=0$.

During the fifth phase, the price path is a pure Hotelling path, $p_{t}=c_{x}+\lambda_{0} e^{\rho t}$, but with part $\bar{y}$ of the energy consumption supplied by the renewable resource. The contribution from non-renewable energy $d_{n}\left(p_{t}\right)$ decreases to zero at the end of the phase, so the price $p_{t_{5}}$ is equal to $\bar{p}_{y}$. At this time, coal is exhausted.

The last phase $\left[t_{5}, \infty\right)$ is the usual phase of renewable energy consumption: $p_{t}=\bar{p}_{y}$, $x_{t}=0$ and $y_{t}=\bar{y}$.

Note that now a rent has to be borne by the user of the renewable energy, starting from 0 at $t=t_{2}$ and increasing up to $\bar{p}_{x y}-c_{y}$ at $t=t_{3}$, constant at this value during the fourth phase $\left[t_{3}, t_{4}\right)$, increasing again up to $\bar{p}_{y}-c_{y}$ during the fifth phase $\left[t_{4}, t_{5}\right)$, and then afterwards remaining constant at this level.

Such a path is characterized by values of the eight variables $\lambda_{0}, \mu_{0}, \eta_{0}$ and $t_{1}$ to $t_{5}$, obtained by solving a system of eight equations similar to the preceding systems.

\subsection{Optimal paths for beginning use of the renewable substitute before sequestration}

Figure 6 illustrates the case in which implementation of the renewable resource must begin before resorting to sequestration, assuming that the reservoir is small.

Figure 6 here 
As in the preceding case, the optimal path consists of six phases. During the first phase $\left[0, t_{1}\right)$, only coal is used and $p_{t}=c_{x}+\lambda_{0} e^{\rho t}-\mu_{0} e^{(\alpha+\rho) t} \zeta, q_{t}=x_{t}=d_{n}\left(p_{t}\right)=d\left(p_{t}\right)$. At the end of the phase, $p_{t}=c_{y}$ so the renewable substitute becomes competitive while the ceiling is slackened, $Z_{t_{1}}\left(\lambda_{0}, \mu_{0}\right)<\bar{Z}$.

During the second phase $\left[0, t_{1}\right)$, both coal and solar energy are being used, while the expression of the price remains the same because the pollution stock $Z_{t}$ lies below $\bar{Z}$, but now with $x_{t}=d_{n}\left(p_{t}\right)<d\left(p_{t}\right)$ and $y_{t}=\bar{y}$. At the end of this phase, the pollution constraint becomes binding and $c_{x}+\lambda_{0} e^{\rho t_{2}}-\mu_{0} e^{(\alpha+\rho) t_{2}} \zeta=c_{x}+\lambda_{0} e^{\rho t_{2}}+\left(c_{s}-\eta_{0} e^{\rho t_{2}}\right) \zeta$. The last four phases are similar to the last four of the preceding case.

It is possible to have paths that involve using the renewable resource immediately at time $t=0$. These would correspond to optimal paths where the initial values $X^{0}$ and $Z^{0}$ of the state variables $X_{t}$ and $Z_{t}$ would be equal to their values at $t^{\prime} \in\left[t_{1}, t_{2}\right)$ following the path illustrated in Figure 6. The argument for this scenario is based on the same concept of time consistency developed in sub-section 5.1.

\section{Conclusion}

We stress the potential role of carbon sequestration in allowing an environmental policy to maintain the atmospheric carbon concentration below some threshold level. We show that, whatever the sink capacity, sequestration should be implemented once the pollution ceiling is reached. In addition, our analysis suggests that the polluting fossil fuel will be exhausted in a finite time, after which the market will be supplied by the renewable substitute $^{16}$, whether or not its capacity is constrained. However, in contrast with the other cases, when the capacity of the renewable resource flow is constrained, and is initially very affordable, the renewable resource must be used before the ceiling is reached. In this latter scenario, the renewable resource could be regarded as a mid-term option for alleviating pollution, while sequestration allows for further emission reductions on the longer term. But, more generally, the lack of sequestration before the ceiling is reached should not be seen as weakening the preventive short-term role of sequestration usually advocated as an

\footnotetext{
${ }^{16}$ This resource exploitation and sequestration scheme, obtained with constant average costs, is robust to alternative specifications for the cost functions. As far as the sequestration or extraction costs are concerned (see Lafforgue et al., 2005, who also studied the case of multiple sequestration sinks), these specifications may depend upon the cumulative sequestered carbon or the cumulative extracted fossil resource, respectively, as in Heal (1976).
} 
option for such a climate-change mitigation. Indeed, whether or not the renewable resource is scarce, the optimal environmental policy affects the extraction of the exhaustible resource anyway, with extraction decreasing until the pollution ceiling is reached. This reduction in consumption should be attributed to the opportunity cost of emitting one unit of carbon before the ceiling, as well as the opportunity cost of sequestering one unit once at the ceiling, these costs being added to the overall exploitation cost of the resource.

Finally, it is noteworthy that our model does not consider the possibility of carbon leakage, since geological or even oceanic sinks may only represent temporary storage options (cf. Herzog et al., 2003; Paccala, 2003). This leakage phenomenon, if continuous over time, would have no short-term incidence on the optimal solution. The phase with sequestration on its own would then be extended to the entire pollution ceiling phase: once the storage capacity has been filled, sequestration would simply allow for compensating the leakage at each moment of time. 


\section{Appendix}

Let $p_{t}^{H}=c_{x}+\lambda_{0} e^{\rho t}$ be the Hotelling price path, $\hat{p}_{t}=p_{t}^{H}-\mu_{0} e^{(\alpha+\rho) t} \zeta$ the optimal price path preceding the ceiling and $\tilde{p}_{t}=p_{t}^{H}+\left(c_{s}-\eta_{0} e^{\rho t}\right) \zeta$, the optimal price path followed within the sequestration phase. In the small reservoir case, the complete solution of $(P)$ is ${ }^{17}$ :

$$
x_{t}=\left\{\begin{array}{ll}
d\left(\hat{p}_{t}\right) & , t \in\left[0, t_{1}\right) \\
d\left(\tilde{p}_{t}\right) & , t \in\left[t_{1}, t_{2}\right) \\
\bar{x} & , t \in\left[t_{2}, t_{3}\right) \\
d\left(p_{t}^{H}\right) & , t \in\left[t_{3}, t_{4}\right) \\
0 & , t \in\left[t_{4}, \infty\right)
\end{array}, y_{t}=\left\{\begin{array}{ll}
0 & , t \in\left[0, t_{4}\right) \\
\tilde{y} & , t \in\left[t_{4}, \infty\right)
\end{array}, s_{t}=\left\{\begin{array}{ll}
0 & , t \notin\left[t_{1}, t_{2}\right) \\
\zeta\left[d\left(\tilde{p}_{t}\right)-\bar{x}\right] & , t \in\left[t_{1}, t_{2}\right)
\end{array} .\right.\right.\right.
$$

Associated Lagrange multipliers are:

$$
\begin{aligned}
& \gamma_{s t}=\left\{\begin{array}{ll}
c_{s}+\mu_{0} e^{(\alpha+\rho) t}-\eta_{0} e^{\rho t} & , t \in\left[0, t_{1}\right) \\
0 & , t \in\left[t_{1}, t_{2}\right) \\
c_{s}+\left(p_{t}^{H}-\bar{p}_{x}\right) / \zeta & , t \in\left[t_{2}, t_{3}\right) \\
c_{s} & , t \in\left[t_{3}, \infty\right)
\end{array}, \bar{\gamma}_{s t}=0, t \geq 0\right. \\
& \gamma_{x t}= \begin{cases}0 & , t \in\left[0, t_{4}\right) \\
p_{t}^{H}-c_{y} & , t \in\left[t_{4}, \infty\right)\end{cases} \\
& \gamma_{y t}= \begin{cases}c_{y}-\hat{p}_{t} & , t \in\left[0, t_{1}\right) \\
c_{y}-\tilde{p}_{t} & , t \in\left[t_{1}, t_{2}\right) \\
c_{y}-\bar{p}_{x} & , t \in\left[t_{2}, t_{3}\right) \\
c_{y}-p_{t}^{H} & , t \in\left[t_{3}, t_{4}\right) \\
0 & , t \in\left[t_{4}, \infty\right)\end{cases} \\
& \eta_{t}=\left\{\begin{array}{ll}
\eta_{0} e^{\rho t} & , t \in\left[0, t_{2}\right) \\
0 & , t \in\left[t_{2}, \infty\right)
\end{array} \quad, \nu_{S t}=0, t \geq 0\right. \\
& \mu_{t}= \begin{cases}\mu_{0} e^{(\alpha+\rho) t} & , t \in\left[0, t_{1}\right) \\
\eta_{0} e^{\rho t}-c_{s} & , t \in\left[t_{1}, t_{2}\right) \\
\left(p_{t}^{H}-\bar{p}_{x}\right) / \zeta & , t \in\left[t_{2}, t_{3}\right) \\
0 & , t \in\left[t_{3}, \infty\right)\end{cases} \\
& \nu_{Z t}=\left\{\begin{array}{ll}
0 & , t \in\left[0, t_{1}\right) \\
(\alpha+\rho) c_{s}-\alpha \eta_{0} e^{\rho t} & , t \in\left[t_{1}, t_{2}\right) \\
{\left[\dot{p}_{t}^{H}-(\alpha+\rho)\left(p_{t}^{H}-\bar{p}_{x}\right)\right] / \zeta} & , t \in\left[t_{2}, t_{3}\right) \\
0 & , t \in\left[t_{3}, \infty\right)
\end{array} .\right.
\end{aligned}
$$

Given (30), it is easy to check that if $\lambda_{0}, \mu_{0}, \eta_{0}, t_{1}, t_{2}, t_{3}$ and $t_{4}$ satisfy the system

\footnotetext{
${ }^{17}$ For the large reservoir case, just set $\eta_{0}=0$.
} 
of seven equations described in section 4 (subsection 4.3), then the Lagrange multipliers defined by (31)-(36) are such that conditions (7)-(19) hold. In other respects, since the non-renewable resource stock $X_{t}$ is exhausted at $t_{4}$, the transversality condition (20) is satisfied. In the same way, since $\eta_{t}=0$ and $S_{t}=\bar{S}$ for $t \geq t_{2}$ on the one hand, $\mu_{t}=0$ and $Z_{t}<\bar{Z}$ for $t \geq t_{4}$ on the other hand, then conditions (21) and (22) are valid. 


\section{References}

[1] Chakravorty, U., Magné B., Moreaux M., 2004. Plafond de concentration atmosphérique en carbone et substitutions entre ressources énergétiques, forthcoming in Annales d'Economie et de Statistique.

[2] Chakravorty, U., Magné B., Moreaux M., 2005a. Transitional dynamics from fossil to bio-fuel under a ceiling pollution constraint, mimeo LERNA, Toulouse University.

[3] Chakravorty, U., Magné B., Moreaux M., 2005b. A Hotelling model with a ceiling on the stock of pollution, forthcoming in Journal of Economic Dynamics and Control.

[4] Edmonds J., Clarke J., Dooley J., Kim S.H., Smith S.J., 2004. Stabilization of $\mathrm{CO}_{2}$ in a B2 world: insights on the roles of carbon capture and disposal, hydrogen, and transportation technologies, Energy Economics, 26(4), 517-537.

[5] Gitz V., Ambrosi P., Magné B., Ciais P., 2005. Is There an Optimal Timing for Sequestration to Stabilize Future Climate? American Geophysical Union, Geophysical Monograph, The Science and Technology of Carbon Sequestration: Assessment and Verification of Natural and Deliberate Carbon Sinks, American Geophysical Union Ed.

[6] Ha Duong, M., Keith, D.W., 2003. Carbon storage: the economic deficiency of storing CO2 in leaky reservoirs, Clean Technologies and Environmental Policy, 5, 181-189.

[7] Heal G., 1976. The Relationship Between Price and Extraction Cost for a Resource with a Backstop Technology, Bell Journal of Economics, The RAND Corporation, 7(2), 371-378.

[8] Herfindahl, O.L., 1967. Depletion and economic theory, in M. Gaffney (ed.), Extractive Resources and Taxation, pp. 63-90: University of Wisconsin Press, Madison.

[9] Herzog, H., Caldeira K., Reilly, J.M., 2003. An issue of permanence: assessing the effectiveness of temporary carbon storage, Climatic Change, 59, 293-310.

[10] Hotelling, H., 1931. The economics of exhaustible resources, Journal of Political Economy, 39, 137-175. 
[11] Kolstad, C.D., Krautkraemer J.A., 1993. Natural resource use and the environment, in A.V. Kneese and J.L. Sweeney (eds.) Handbook of Natural Resource and Energy Economics, Vol. III, Chap. 26: Elsevier Science Publishers.

[12] Kurosawa, A., 2004. Carbon concentration target and technological choice, Energy Economics, 26(4), 675-684.

[13] Lafforgue, G., Magné B., Moreaux, M., 2005. Substitutions entre effet de serre et puits de carbone, mimeo LERNA, Toulouse University.

[14] McFarland, J.R., Herzog, H.J., Reilly, J.M., 2003. Economic modeling of the global adoption of carbon capture and sequestration technologies, in J. Gale and Y. Kaya (eds.), Greenhouse Gas Control Technologies: Proceedings of the Sixth International Conference on Greenhouse Gas Control Technologies: Elsevier Science, Oxford, UK.

[15] Pacala, S.W., 2003. Global Constraints on Reservoir Leakage, in J. Gale and Y. Kaya (eds.), Greenhouse Gas Control Technologies: Proceedings of the Sixth International Conference on Greenhouse Gas Control Technologies: Elsevier Science, Oxford, UK.

[16] Riahi, K., Rubin E.S., Taylor M.R., Schrattenholzer L., Hounshell, D., 2004. Technological learning for carbon capture and sequestration technologies, Energy Economics, 26(4), 539-564.

[17] Tahvonen, O., 1997. Fossil fuels, stock externalities and backstop technology, Canadian Journal of Economics, 30(4), 855-874.

[18] Tahvonen, O., Salo, S., 1996. Nonconvexities in optimal pollution accumulation, Journal of Environmental Economics and Management, 31(2), 160-177.

[19] Tahvonen, O., Withagen, C., 1996. Optimality of irreversible pollution accumulation, Journal of Economic Dynamics and Control, 20(9-10), 1775-1795.

[20] Toman, M.A., Withagen, C., 1996. Accumulative pollution, "clean technology" and policy design, Resource and Energy Economics, 22(4), 367-384. 


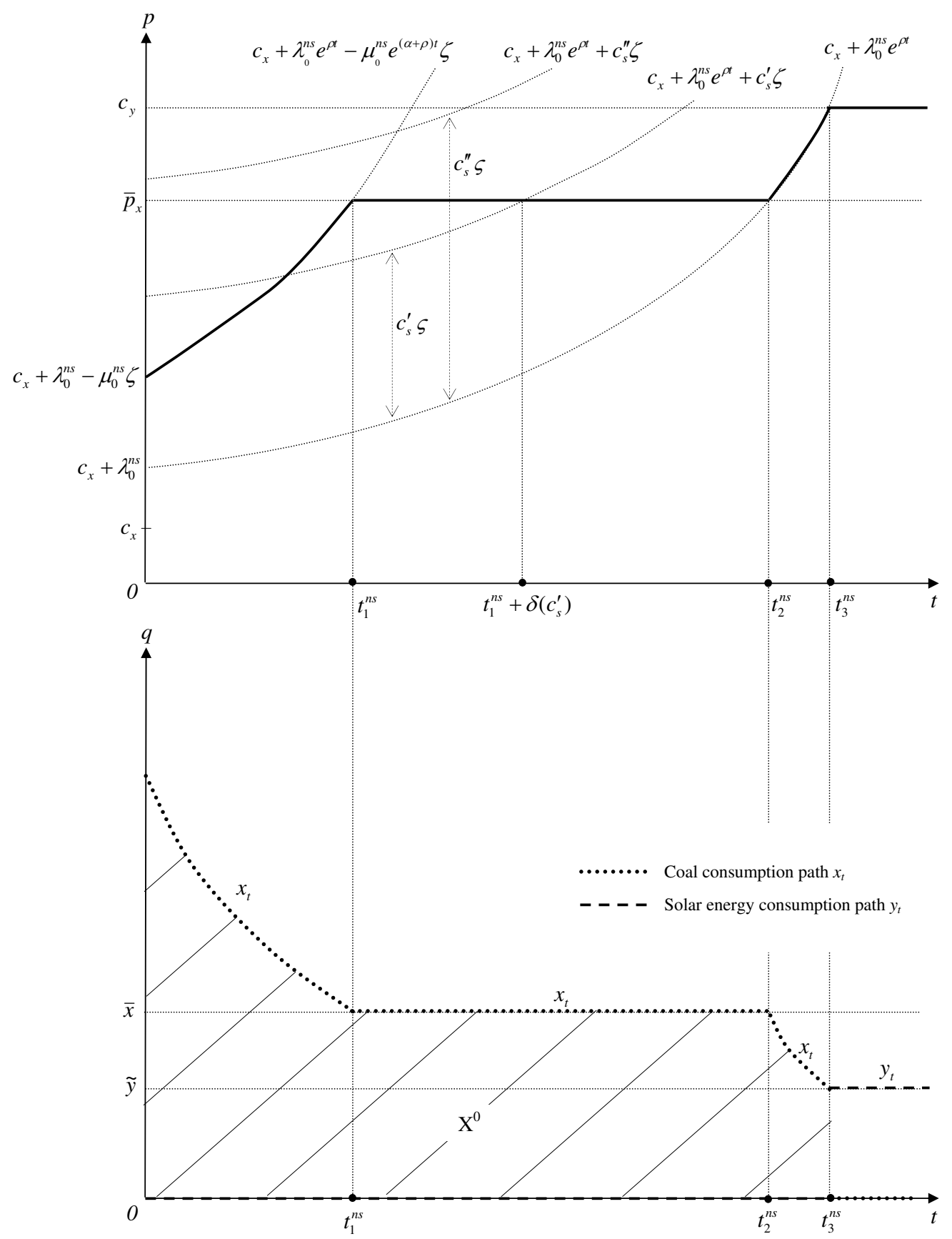

Figure 1: Optimal paths without any sequestration opportunity 


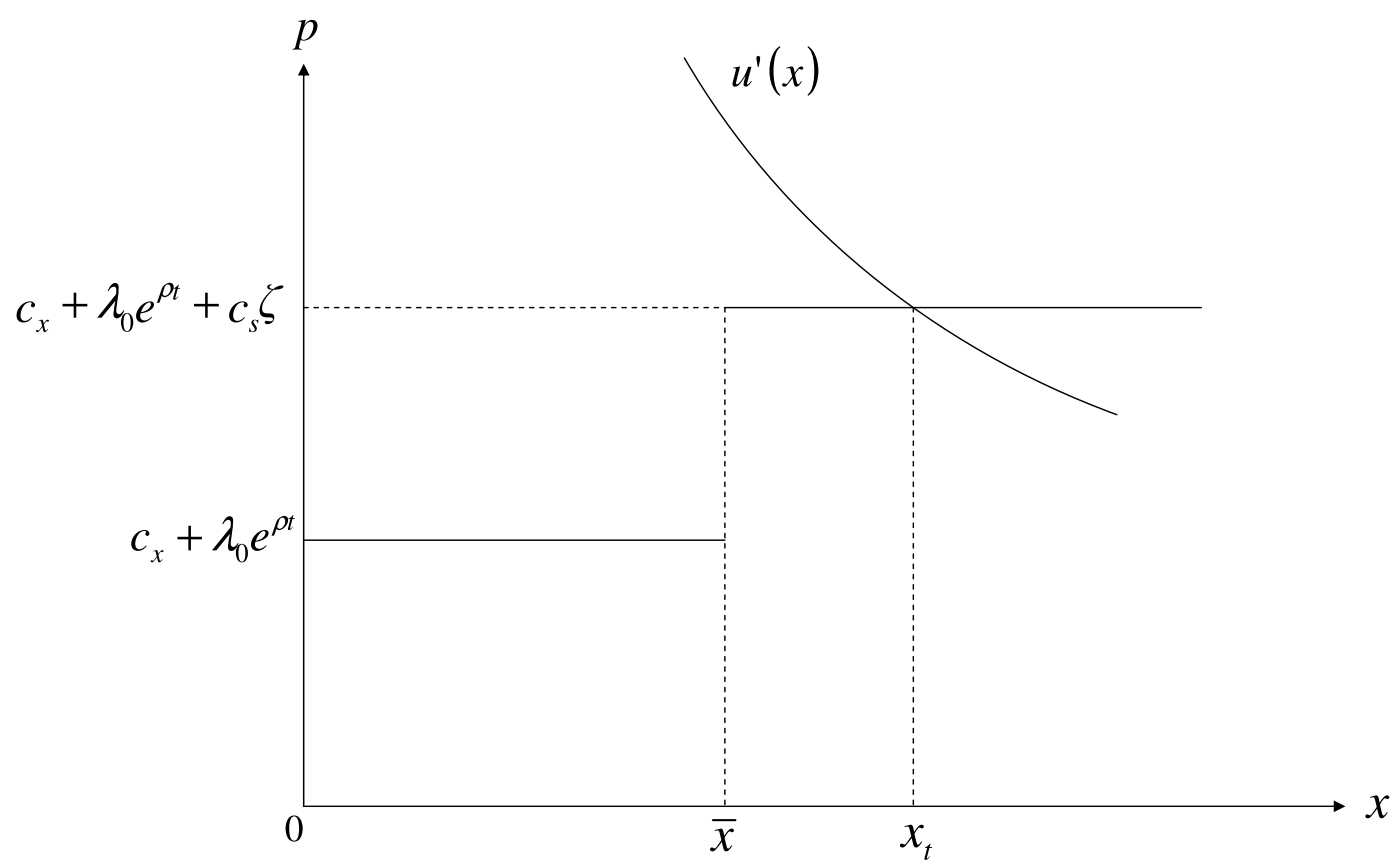

Figure 2: Determination of the optimal consumption rate of coal during the sequestration phase 


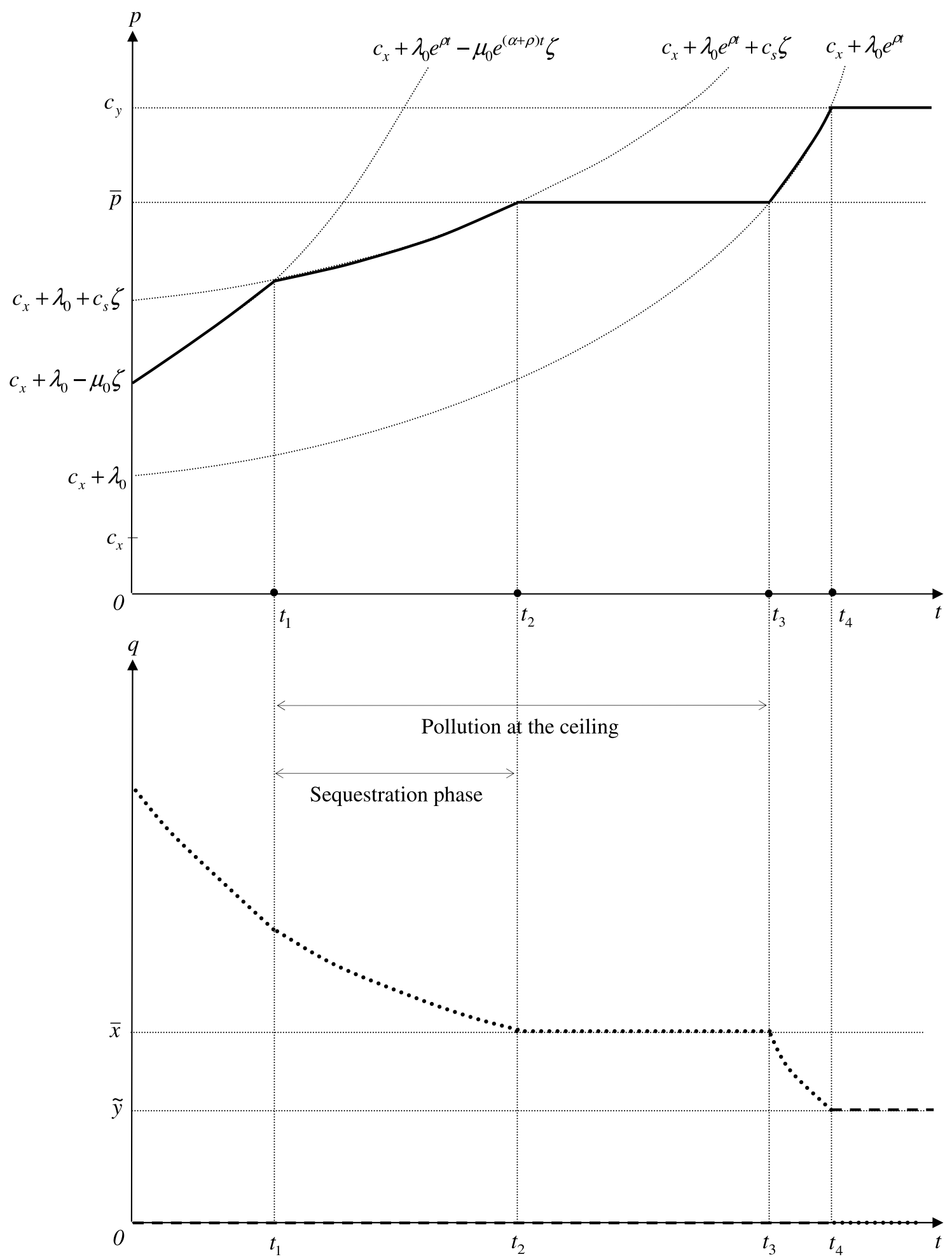

Figure 3: Optimal paths - The large reservoir case 


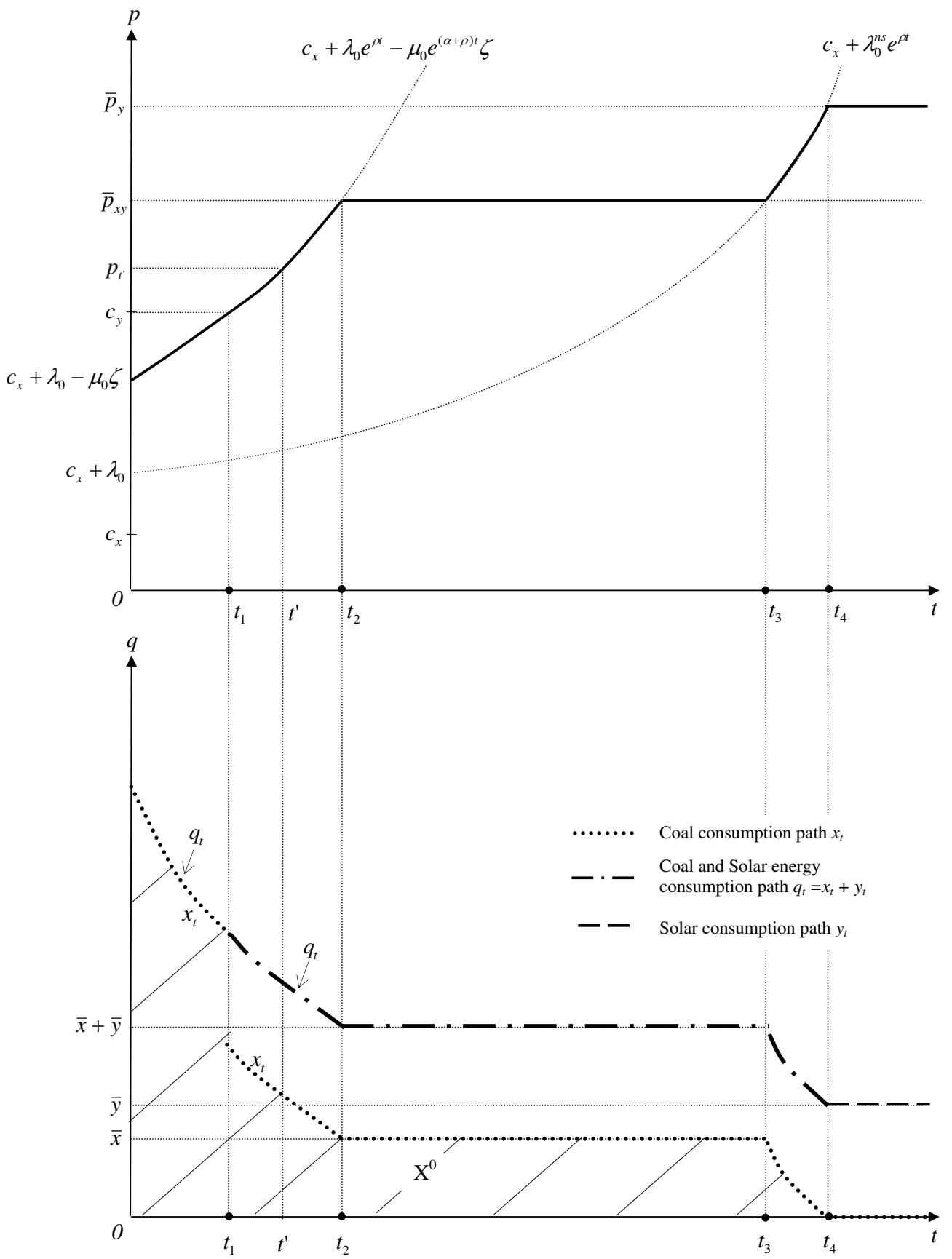

Figure 4: Optimal paths - No sequestration allowed, rare renewable substitute 


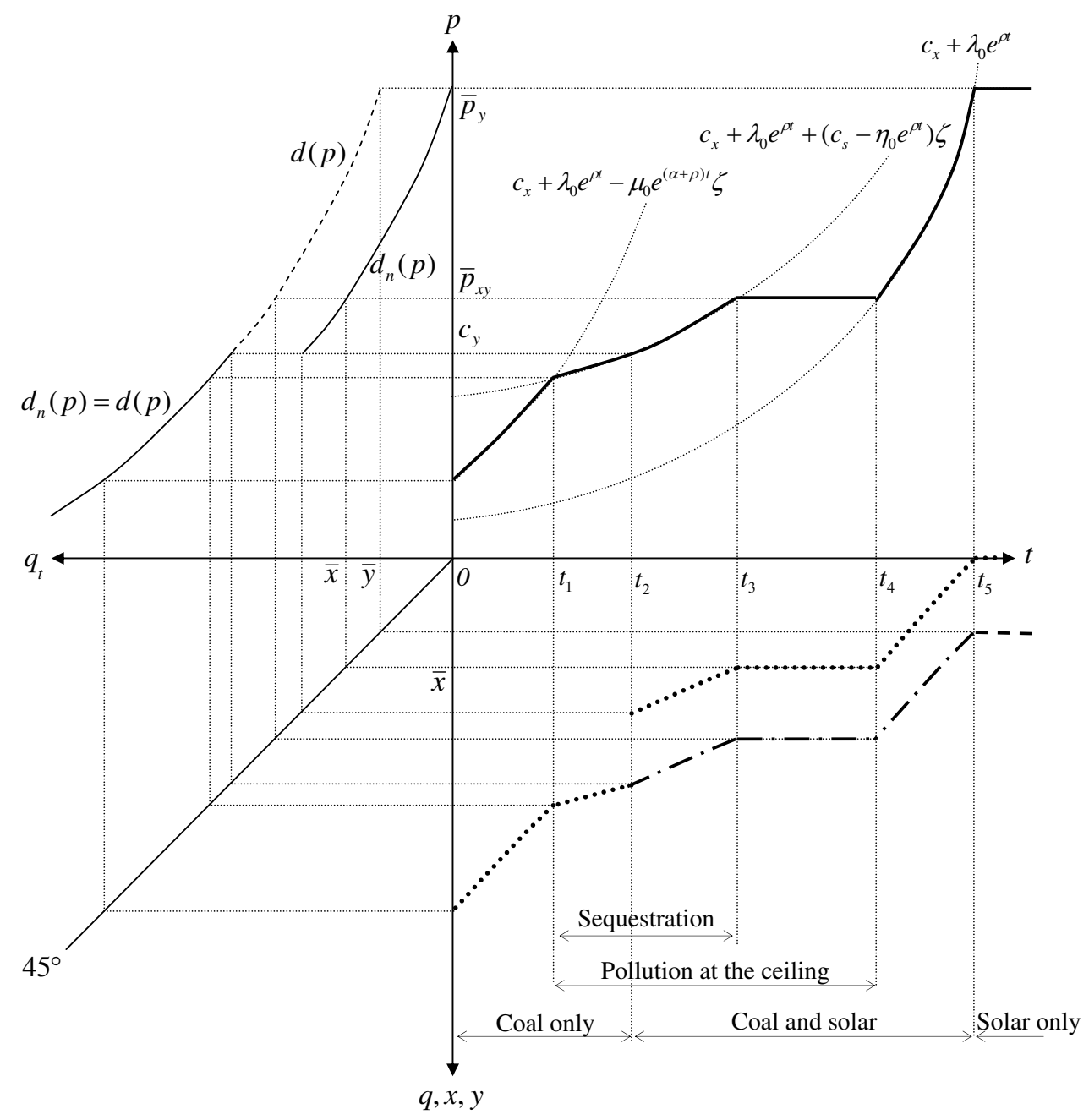

Figure 5: Optimal paths with a rare renewable resource. Sequestration implemented before the renewable substitute 


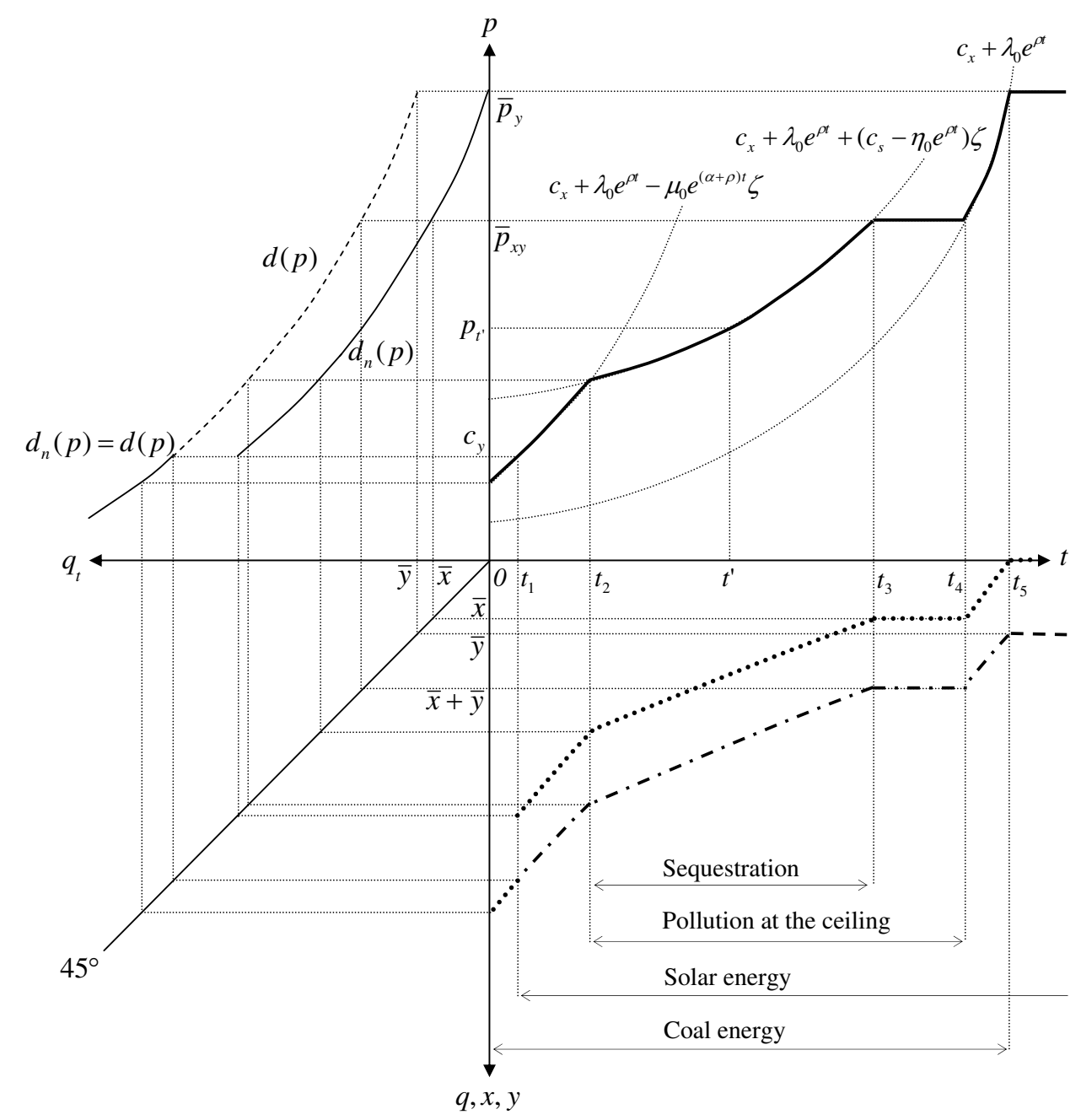

Figure 6: Optimal paths with a rare renewable resource. Sequestration implemented after the renewable substitute 